\title{
Dense Ceramic Membranes for Hydrogen Separation
}

Truls Norby and Reidar Haugsrud

\section{1}

Introduction

In the 1980s and 1990s, the development of oxygen ion conductors for solid electrolytes, as well as mixed oxygen-electron conductors as electrodes for solid oxide fuel cells, brought scientists to envision a possibility of mixed-conducting oxygenpermeable ceramic membranes. Today this field is well established, and ceramic membranes are close to implementation in processes for oxygen extraction from air as well as for direct partial oxidation of natural gas. The technology seems to offer superior routes for efficient power production from fossil fuels, combined with $\mathrm{CO}_{2}$ sequestration. Many materials with very high oxygen ion and electronic conductivities are available.

It was not equally obvious that dense ceramic hydrogen-permeable membranes would be of similar interest. There are clearly needs for hydrogen purification membranes, but polymers and microporous materials as well as metals such as palladium and its alloys appeared to fill these needs. In addition, possible candidates for dense ceramic hydrogen-permeable materials were not as appealing as the oxygen-permeable ones in terms of performance and stability.

As plans for fossil-fuel-based power plants incorporating oxygen-permeable membranes were developed, it became clear that hydrogen-permeable membranes might find use as well. In particular, this would be the case if the temperature of operation was high enough that hydrogen-permeable ceramic membranes could be thermally integrated with other processes, such as reforming, oxygen separation by membranes, gas turbines, or solid oxide fuel cells. Several research groups and industries have therefore focused on the possibility of developing materials and related technologies for ceramic hydrogen-permeable membranes.

At present, the known hydrogen-permeable dense ceramic materials are oxides that are mixed proton-electron conductors. We would claim that ceramic hydrogen-separation membranes are in most aspects more challenging than their oxygen-permeable counterparts. Proton transport at high temperatures is fast, but thermodynamics speaks against a high concentration of protons in the materials at high temperatures. Combinations of both high protonic and electronic conduc- 
tivity appear to be remarkably rare. Doping strategies, which enhance the concentration of protons, mostly counteract the desired n-type electronic conductivity [1]. The addition of hydrogen defects makes the defect chemistry more complex. Characterization of hydrogen permeation itself is more difficult as there are more sources of error and misinterpretation. It has been suggested that diffusion of neutral hydrogen species can be a major contributor to hydrogen permeation not only in metals, but also in ceramics, to a larger extent than formerly anticipated [2]. Finally, framework materials with sub-nano-sized cages appear to provide interesting borderline cases between microporous hydrogen permeation and mixed ionic-electronic conduction [3].

This chapter aims to keep these challenges in mind as we review the defect chemistry, transport theory and aspects of characterization of hydrogen permeation in dense ceramics. We will first look at some applications and simple schemes of operation of hydrogen-permeable membranes and then, briefly, at the literature and status of hydrogen-permeable dense ceramics.

\section{2}

\section{Applications and Principles of Operation}

\subsection{1}

\section{Simple Cases}

In the following, we examine some applications and the principles involved. Processes may have different conditions that establish the driving forces and fluxes of hydrogen and other species in different ways. In addition to hydrogen, it is of particular interest to look at driving forces for oxygen transport, because many dense ceramic hydrogen membranes also conduct oxygen ions.

Hydrogen-separation membranes extract hydrogen from mixtures of gases using a hydrogen partial pressure gradient as the driving force. This can be achieved using high pressure on the feed side or low pressure on the permeate side. Figure 1.1 shows hydrogen separation from nitrogen as an example.

a)

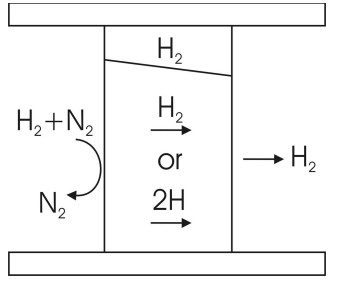

b)

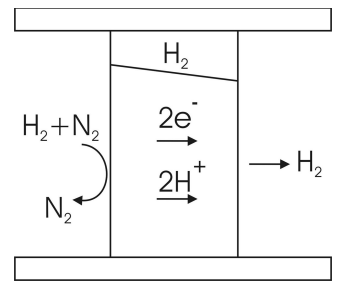

Figure 1.1 Schematic representation of hydrogen permeable membranes. Separation from a mixture with nitrogen is used as an example. The driving force is provided by a high total pressure on the feed side or pump- ing on the permeate side. Gradient in hydrogen chemical potential is illustrated qualitatively.

(a) Permeation of molecules (as in porous materials) or neutral atoms (as in metals).

(b) Mixed proton-electron conductor. 
Figure 1.1a shows schematically the operation of a membrane that is permeable to hydrogen molecules (corresponding to a porous membrane or a dense material in which molecules dissolve and diffuse) or to neutral hydrogen atoms (corresponding to a material in which hydrogen dissolves dissociatively, as in a metal). Figure 1.1b shows schematically how a mixed proton-electron conductor performs the same process by so-called ambipolar diffusion of both protons and electrons in the same direction to maintain electroneutrality and zero net current.

High-temperature membranes allow hydrogen separation to accompany and be thermally integrated with chemical reactions. First and foremost - and always central in equilibria with oxidic membranes - is the equilibrium between hydrogen, oxygen, and water vapor Eq. (1.1):

$$
\mathrm{H}_{2}(\mathrm{~g})+\frac{1}{2} \mathrm{O}_{2}(\mathrm{~g})=\mathrm{H}_{2} \mathrm{O}(\mathrm{g}) ; \quad K_{\mathrm{H}_{2} \mathrm{O}}=\frac{p_{\mathrm{H}_{2} \mathrm{O}}}{p_{\mathrm{H}_{2}} p_{\mathrm{O}_{2}}^{1 / 2}}=\exp \frac{\Delta S_{\mathrm{H}_{2} \mathrm{O}}^{0}}{R} \exp \frac{-\Delta H_{\mathrm{H}_{2} \mathrm{O}}^{0}}{R T}
$$

where $K_{\mathrm{H}_{2} \mathrm{O}}$ is the equilibrium constant, $p_{\mathrm{H}_{2} \mathrm{O}}, p_{\mathrm{H}_{2}}$ and $p_{\mathrm{O}_{2}}$ are partial pressures, $\Delta S_{\mathrm{H}_{2} \mathrm{O}}^{0}$ and $\Delta H_{\mathrm{H}_{2} \mathrm{O}}^{0}$ are the standard entropy and enthalpy changes, $R$ is the ideal gas constant, and $T$ the absolute temperature. At room temperature, we have $\Delta S_{\mathrm{H}_{2} \mathrm{O}, 298}^{0}=-44.42 \mathrm{~J} \mathrm{~mol}^{-1} \mathrm{~K}^{-1}$ and $\Delta H_{\mathrm{H}_{2} \mathrm{O}, 298}^{0}=-241.83 \mathrm{~kJ} \mathrm{~mol}^{-1}$, while at $1000^{\circ} \mathrm{C}$ they are $\Delta S_{\mathrm{H}_{2} \mathrm{O}, 1273}^{0}=-56.63 \mathrm{~J} \mathrm{~mol}^{-1} \mathrm{~K}^{-1}$ and $\Delta H_{\mathrm{H}_{2} \mathrm{O}, 1273}^{0}=-249.40 \mathrm{~kJ} \mathrm{~mol}^{-1}$. For example, hydrogen may be extracted through a membrane by reaction with oxygen in air according to the above equilibrium. This creates a high chemical driving force. Although the product is simply water vapor (steam), the process may be of interest if the oxygen-depleted permeate (here nitrogen + steam) can be used elsewhere and if the heat generated can be supplied to another reaction which requires it. Such a reaction is the endothermic steam reforming of methane, as shown schematically in Fig. 1.2a.

Figure $1.2 \mathrm{~b}$ represents the same dehydrogenation of methane, but using an inert sweep gas (here $\mathrm{N}_{2}$ as an example) instead of oxidation. It should be noted

a)

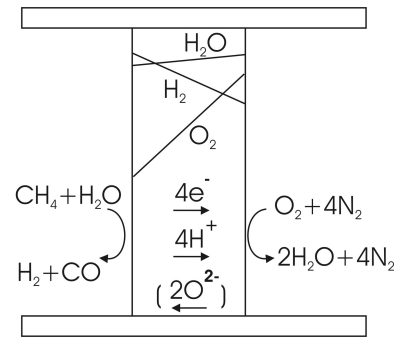

Figure 1.2 Schematic representation of operation, gradients, and fluxes in mixed proton-electron-conducting membranes used for dehydrogenation of reformed methane (syngas). Gradients represent qualitatively chemi- b)

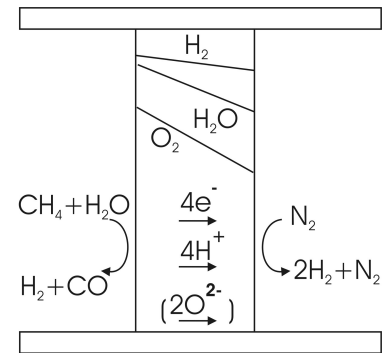

cal potentials of $\mathrm{H}_{2}, \mathrm{O}_{2}$, and $\mathrm{H}_{2} \mathrm{O}$. Flux in parentheses represents oxygen flux in the case of non-negligible oxygen ion transport number. (a) Use of oxygen in air to create a large gradient. (b) Use of sweep gas (here $\mathrm{N}_{2}$ ). 
that the wet nitrogen output from the membrane in Fig. 1.2a could be used for a stage operated like the membrane in Fig. 1.2b.

In Fig. 1.2, oxygen activity gradients are indicated. If the transport number of oxygen ions is significant, the hydrogen flux can be accompanied by an oxygen flux. If hydrogen and oxygen ions flow in the same direction (as would be the case in Fig. 1.2b), water appears in the permeate along with the hydrogen. If they flow in opposite directions (as in Fig. 1.2a), the oxygen flux adds to the oxidation of the reformed methane and depletion of oxygen on the air side. The latter indicates that mixed proton, oxygen ion, and electron conduction can be useful.

\section{2 .2}

\section{Examples of More Complex Applications}

The utilization of hydrogen-separation membranes in large-scale chemical processing or in fossil-fueled power plants may be enhanced by combining more than one stage and by integration with other types of devices, such as heat exchangers, catalytic reactors, gas turbines, or fuel cells. Figure 1.3 shows schematically an example of the combined use of oxygen- and hydrogen-permeable membranes.

Figure 1.4 shows a sketch of processes utilizing two hydrogen-separation stages, one step for deoxidizing air using a reforming methane-steam mixture, and one

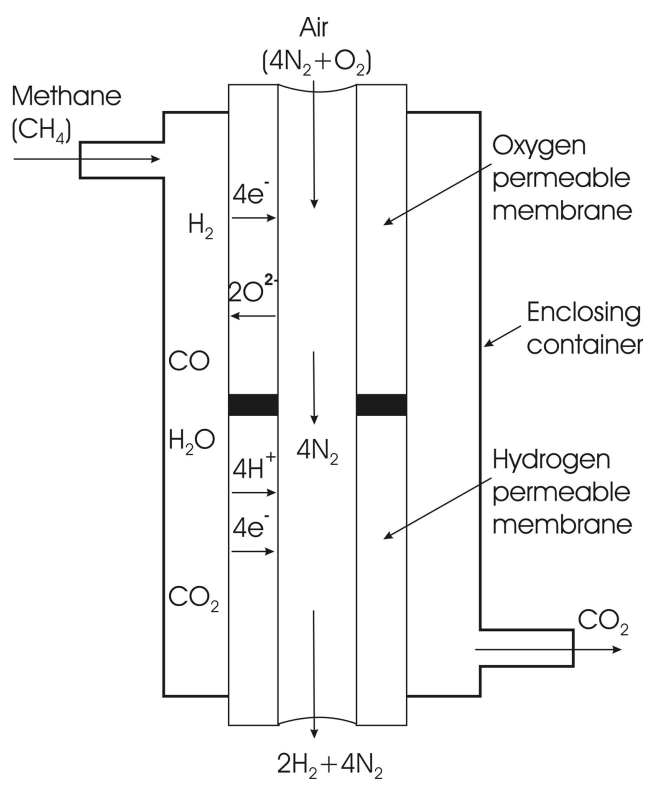

Figure 1.3 Schematic illustration of sequential use of a mixed oxygen ion electron conductor for oxygen separation (upper tube) and mixed proton-electron conductor for hydrogen separation. The air flowing inside the tubes becomes an $\mathrm{H}_{2}+\mathrm{N}_{2}$ mixture ready for a fuel cell or gas turbine. The methane flowing in the outer chamber is transformed into $\mathrm{CO}_{2}$, which can be dried and compressed for sequestration. 


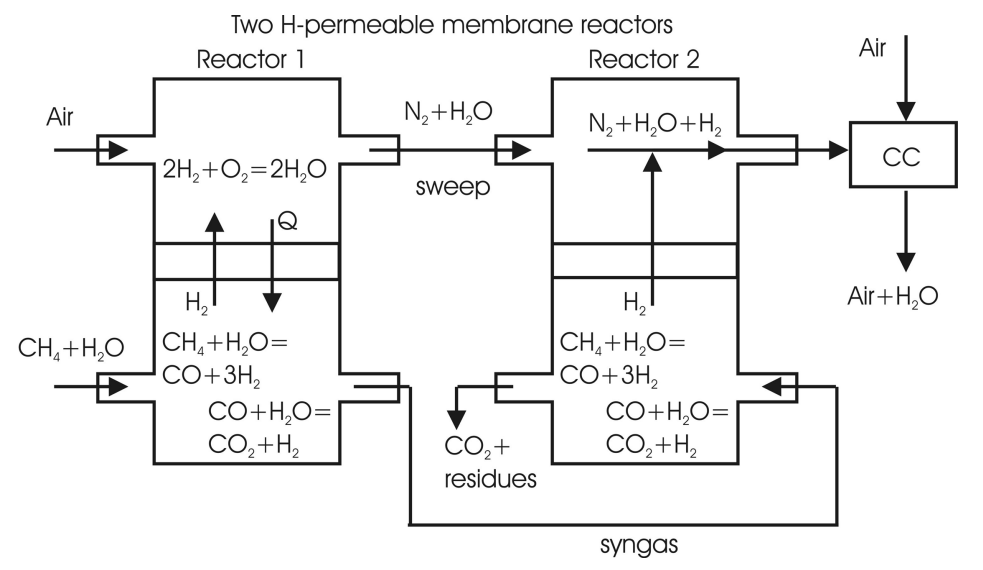

Figure 1.4 Schematic of an example of the use of two $\mathrm{H}$-permeable membrane reactors in a natural gas-fired power plant with $\mathrm{CO}_{2}$ sequestration. Reactor 1 consumes $\mathrm{O}_{2}$ in an air stream and exchanges the heat evolved (Q) with the reforming reaction. Reactor 2

sweeps $\mathrm{H}_{2}$ out of the syngas using the depleted air as sweep gas and supplies it to a gas turbine. Reactors 1 and 2 are shown in co-flow and counter-flow configurations, respectively. Adapted from Åsen et al. [4].

for using the resulting $\mathrm{N}_{2}+\mathrm{H}_{2} \mathrm{O}$ for sweeping hydrogen out of the reformate, as suggested by Åsen et al. [4].

However, such systems are not realized at any significant scale at present (2006).

\section{3}

\section{Defect Chemistry of Dense Hydrogen-permeable Ceramics}

\subsection{1}

\section{Materials Classes}

Although metals are relatively permeable to hydrogen at elevated temperatures, few of them are stable at the high temperatures and under the chemical reaction conditions of interest to us here. Metals will thus not be discussed in this chapter, and we restrict ourselves to ceramics, to explore their characteristics and principles of operation.

In principle, metal hydrides, such as $\mathrm{YH}_{3}$, or metal oxyhydrides such as $\mathrm{LaHO}$ or NdHO, may be hydrogen-permeable by diffusion of atoms or molecules or by mixed hydride ion and electron conduction. However, operating conditions of practical interest are (by far) not reducing enough that materials containing hydride ions $\left(\mathrm{H}^{-}\right)$are stable, and we thus also disregard these materials in the following discussion.

In principle, a hydroxide or oxyhydroxide or other compound containing stoichiometric amounts of hydrogen in an oxidation state of +1 could also be used. 
However, no such material is known in which mixed proton-electron conduction is sufficiently high in the solid state at high temperatures. Therefore, in effect, the materials that we will consider are all nominally hydrogen-free. They are compounds - hitherto exclusively oxides - that dissolve hydrogen defects in equilibrium with the surrounding process gases. We now concentrate on the defects that give rise to hydrogen transport in these materials. Later we derive expressions for the flux through membranes of the materials.

\section{3 .2}

\section{Neutral and lonized Hydrogen Species in Oxides}

Membrane materials will be exposed to a hydrogen activity from hydrogen-containing gases, such as $\mathrm{H}_{2}$ and $\mathrm{H}_{2} \mathrm{O}$. At equilibrium, it is sufficient to consider only one of them and to use the equilibrium between them (Eq. (1.1)) as necessary. Hydrogen may dissolve in membrane materials as interstitial atoms or as molecules. As long as the concentration of hydrogen is low and the availability of interstitial sites is high, we can write:

$$
\begin{array}{lll}
\mathrm{H}_{2}(\mathrm{~g})=2 \mathrm{H}_{\mathrm{i}} & K_{2 \mathrm{H}}=\frac{\left[\mathrm{H}_{\mathrm{i}}\right]^{2}}{p_{\mathrm{H}_{2}}} ; & {\left[\mathrm{H}_{\mathrm{i}}\right]=K_{2 \mathrm{H}}^{1 / 2} p_{\mathrm{H}_{2}}^{1 / 2}=K_{\mathrm{S}} p_{\mathrm{H}_{2}}^{1 / 2}} \\
\mathrm{H}_{2}(\mathrm{~g})=\left(\mathrm{H}_{2}\right)_{\mathrm{i}} & K_{\mathrm{H}_{2}}=\frac{\left[\left(\mathrm{H}_{2}\right)_{\mathrm{i}}\right]}{p_{\mathrm{H}_{2}}} ; & {\left[\left(\mathrm{H}_{2}\right)_{\mathrm{i}}\right]=K_{\mathrm{H}_{2}} p_{\mathrm{H}_{2}}}
\end{array}
$$

and the equilibrium concentrations of the hydrogen species are then given by these equations independent of other defects and electroneutrality. It may be noted that Eq. (1.2) gives the well-known Sieverts' law behavior, for which the dissolved hydrogen concentration is proportional to the square root of the hydrogen partial pressure. The proportionality constant is called Sieverts' constant, $K_{\mathrm{S}}$, and equals the square root of the equilibrium constant, $K_{2 \mathrm{H}}$.

The concentration of these dissolved species and their ionization to $\mathrm{H}^{+}$and $\mathrm{H}^{-}$ are of interest in semiconductor physics because many of the hydrogen species may act as terminators for various defects. In this way, the number of defects active as recombination centers is reduced. It has recently been proposed that the ground state energy level of the $\mathrm{H}^{0}$ electron relative to the vacuum level is largely the same (around $-4 \mathrm{eV}$ ) in all oxides and other semiconductors [5]. Thus, ionization of neutral hydrogen to release an electron $\left(\mathrm{H}^{0}=\mathrm{H}^{+}+\mathrm{e}^{-}\right)$within the solid is dependent on the position of the conduction band. In some oxides, such as $\mathrm{ZnO}$, hydrogen is expected to be a so-called shallow donor and ionize easily to $\mathrm{H}^{+}$, whereas in others, such as $\mathrm{MgO}, \mathrm{Al}_{2} \mathrm{O}_{3}$, and $\mathrm{ZrO}_{2}$, the hydrogen is a so-called deep donor and may largely remain unionized. Aliovalent doping or reduction or oxidation obviously change the Fermi level and affect the degree of ionization.

However, it is important to note a major difference between the considerations in semiconductor physics and in high temperature chemistry: In semiconductors, 
the hydrogen is present from the fabrication or is inserted, for example, by ion bombardment or by thermal in-diffusion. Thereafter, the hydrogen is largely considered immobile and at constant total concentration distributed between $\mathrm{H}^{-}, \mathrm{H}^{0}$, and $\mathrm{H}^{+}$states. In high-temperature electrochemistry, on the other hand, the hydrogen species in the solid are in equilibrium with the gas phase according to the equations above. Thus, a particular material at a given temperature and partial pressure of molecular hydrogen, $p_{\mathrm{H}_{2}}$, will have a given concentration of neutral hydrogen species irrespective of doping or Fermi level of the oxide. The latter will surely affect the concentration of ionized species, but the former equilibria will ascertain that the concentrations of neutral species are at their equilibrium levels.

The equilibrium concentrations of hydrogen atoms and molecules in oxides are perhaps not widely different between different oxides. As a first estimate, the entropy of the reaction from one mole of $\mathrm{H}_{2}$ gas to $\left(\mathrm{H}_{2}\right)_{\mathrm{i}}$ or $2 \mathrm{H}_{\mathrm{i}}$ in the solid may be expected to be $-120 \mathrm{~J} \mathrm{~mol}^{-1} \mathrm{~K}^{-1}$, implying that at very high temperatures (entropy controlled) we would have occupied site fractions of the order of $1 \mathrm{ppm}$ of the interstitial sites for $\left(\mathrm{H}_{2}\right)_{\mathrm{i}}$ and $0.1 \%$ for $\mathrm{H}_{\mathrm{i}}$. The enthalpy of dissolution, which determines how the concentrations develop with decreasing temperature, contains the bonding of the $\mathrm{H}$ species in the lattice or to existing defects and - in the case of atoms $\mathrm{H}_{\mathrm{i}}$ - the breaking of the bond $\left(435 \mathrm{~kJ} \mathrm{~mol}{ }^{-1}\right)$ of the $\mathrm{H}_{2}$ molecule. The total may come out positive or negative.

In addition to interstitial dissolution, one may envision hydrogen dissolved substitutionally, that is, in vacancies of oxygen ions or of metal cations. The reasoning for this is that once the site is vacant, there should be little energy cost - perhaps even a gain - in dissolving the neutral hydrogen species there. This hydrogen may be mobile by motion of the vacancy. However, we will not discuss substitutional hydrogen defects further here.

All in all, the equilibrium concentration of neutral hydrogen species will depend on $p_{\mathrm{H}_{2}}$ and temperature, but not on Fermi level, doping or oxygen activity (at constant $p_{\mathrm{H}_{2}}$ ). The concentrations will probably not be very high, but also not very low either. Their temperature dependences are probably not very strong.

The neutral hydrogen species may give rise to considerable hydrogen permeability, a possibility considered by some scientists, as we shall see towards the end of this chapter, but in general not in the gas separation membrane community.

\subsection{4}

\section{Protonic Defects and Their Transport}

Next, we consider the protonic state of hydrogen. A proton is an elementary positive particle with radius of only $10^{-15} \mathrm{~m}\left(10^{5}\right.$ times smaller than any other chemical species). Protons cannot persist in the free state in a chemical environment but are attracted into nearby electron clouds. In oxides and other oxidic materials, attraction will be to oxygen anions to form the hydroxide ion, $\mathrm{OH}^{-}$. As such, the proton residing on a normal oxygen ion can be considered as an interstitial proton, $\mathrm{H}_{\mathrm{i}}{ }^{\bullet}$ (but with the interstitial site strongly displaced towards a particular oxygen ion) or as a substitutional hydroxide ion, $\mathrm{OH}_{\mathrm{O}}^{\bullet}$. 
The predominating transport mechanism for such protons is by free proton jumps (Grotthus mechanism) between neighboring oxygen ions although, statistically, a concentration of oxygen vacancies will enable some protons to move as $\mathrm{OH}^{-}$ions when the host oxygen ions jump to vacancies.

Transport of protons through an oxidic lattice is a complicated process. The effective activation energies range from $30 \mathrm{~kJ} \mathrm{~mol}^{-1}$ for the fastest proton-conducting solid acids such as $\mathrm{CsHSO}_{4}$, and typically $50 \mathrm{~kJ} \mathrm{~mol}^{-1}$ for barium-based perovskite oxides, to $100 \mathrm{~kJ} \mathrm{~mol}^{-1}$ and above for other oxides in which the lattice becomes more densely packed. The proton in most cases spends most of the time rotating around the oxygen ion, forming temporary hydrogen bonds to neighboring oxygen ions. More rarely it jumps over the hydrogen bond to a neighboring oxygen ion. The activation energy for this in a static lattice is high - much higher than the observed activation energies. Dynamic modeling shows that the vibrations (phonons) of the host sublattice are essential to facilitate proton jumps. As two neighboring oxygen ions temporarily move closer during a vibration, the activation energy for proton transfer reduces, allowing the proton to jump over or tunnel through the barrier. For this reason, wide lattices with extensive oxygen dynamics, as in $\mathrm{BaMO}_{3}$ perovskites, have high proton mobilities, while very closepacked, stiff lattices as in $\alpha-\mathrm{Al}_{2} \mathrm{O}_{3}$ have the lowest proton mobilities - despite the shorter nominal O-O-distances in the latter.

According to the above, proton mobilities should be characterized by an activation energy that is dominated by a fraction of the activation energy for oxygen vacancy mobility. This is because the oxygen ion host has only to partly penetrate the energy barrier of approach to a neighboring site, whereas the jump to a vacancy would have required overcoming the full energy barrier. In addition, there remains a small activation energy for the proton transfer. As a rule of thumb, the effective activation energy for proton mobility is $2 / 3$ that of oxygen vacancy mobility. The pre-exponential factor for proton mobility should classically be an order of magnitude higher than for oxygen vacancy mobility because of the low reduced mass and high frequency of the $\mathrm{O}-\mathrm{H}$ oscillator. However, several factors counteract this [6], such as a relatively low "sticking probability" of an otherwise successful proton jump, and the fact that it is the oxygen ion attempt frequency that matters rather than that of the proton.

\subsection{5}

\section{Defect Structures of Proton-conducting Oxides}

Defect-structure-wise there are two main routes to a mixed proton-electron conducting oxide. The simplest would be to dissolve protons compensated by electrons originating directly from hydrogen gas:

$$
\mathrm{H}_{2}(\mathrm{~g})+2 \mathrm{O}_{\mathrm{O}}^{\mathrm{x}}=2 \mathrm{OH}_{\mathrm{O}}^{\bullet}+2 \mathrm{e}^{/} ; \quad K_{2 \mathrm{H} 2 \mathrm{e}}=\frac{\left[\mathrm{OH}_{\mathrm{O}}^{\bullet}\right]^{2} n^{2}}{p_{\mathrm{H}_{2}}\left[\mathrm{O}_{\mathrm{O}}^{\mathrm{x}}\right]^{2}}
$$


This reaction was reported for $\mathrm{ZnO}$ already in the 1950s [7], and it was found that protons and electrons were the dominating defects in hydrogen atmospheres, so that the electroneutrality condition and concentrations are expressed as:

$$
\left[\mathrm{OH}_{\mathrm{O}}^{\bullet}\right]=n=K_{2 \mathrm{H} 2 \mathrm{e}}^{1 / 4} p_{\mathrm{H}_{2}}^{1 / 4}\left[\mathrm{O}_{\mathrm{O}}^{\mathrm{x}}\right]^{1 / 2}
$$

It may be shown that one may only hope to achieve this situation when the material is already dominated by electrons (and some positive point defects, such as oxygen vacancies) under hydrogen-free reducing conditions. By increasing $p_{\mathrm{H}_{2}}$, the native positive defects are replaced by the protonic ones. Protons compensated by electrons are, however, hardly known in systems other than $\mathrm{ZnO}$, and instead one tends to use acceptor-doped oxides in which the concentrations of protons and all other positive defects are enhanced by the doping. The acceptors are most often substitutionally dissolved lower-valent cations, while, in principle, interstitial anions or substitutional higher-valent anions (e.g., $\mathrm{N}^{3-}$ substituting $\mathrm{O}^{2-}$ ) can also be used. We shall simply refer to the acceptors as A, and, in the ionized state, A/.

Assuming that the acceptors are compensated by oxygen vacancies in the absence of hydrogen-containing gases, the reaction with water vapor is given by:

$$
\mathrm{H}_{2} \mathrm{O}(\mathrm{g})+\mathrm{v}_{\mathrm{O}}^{\bullet \bullet}+\mathrm{O}_{\mathrm{O}}^{\mathrm{x}}=2 \mathrm{OH}_{\mathrm{O}}^{\bullet} ; \quad K=\frac{\left[\mathrm{OH}_{\mathrm{O}}^{\bullet}\right]^{2}}{\left[\mathrm{v}_{\mathrm{O}}^{\bullet \bullet}\right]\left[\mathrm{O}_{\mathrm{O}}^{\mathrm{x}}\right] \mathrm{p}_{\mathrm{H}_{2} \mathrm{O}}}=\exp \frac{\Delta S^{0}}{R} \exp \frac{-\Delta H^{0}}{R T}
$$

The equilibrium constant, $K$, for the reaction, and the limiting electroneutrality condition (1.7)

$$
2\left[\mathrm{v}_{\mathrm{O}}^{\bullet \bullet}\right]+\left[\mathrm{OH}_{\mathrm{O}}^{\bullet}\right]=\left[\mathrm{A}^{\prime}\right]=\text { constant }
$$

yield (assuming $\left[\mathrm{v}_{\mathrm{O}}^{\bullet \bullet}\right]+\left[\mathrm{OH}_{\mathrm{O}}^{\bullet}\right]<<\left[\mathrm{O}_{\mathrm{O}}^{\mathrm{x}}\right] \approx[\mathrm{O}]$ ) a simplified expression for $\left[\mathrm{OH}_{\mathrm{O}}^{\bullet}\right]$ :

$$
\left[\mathrm{OH}_{\mathrm{O}}^{\bullet}\right]=\frac{[\mathrm{O}] K p_{\mathrm{H}_{2} \mathrm{O}}\left(-1+\sqrt{1+\frac{8\left[A^{/}\right]}{[\mathrm{O}] K p_{\mathrm{H}_{2} \mathrm{O}}}}\right)}{4}
$$

where $[\mathrm{O}]$ is the concentration of oxygen sites. From this, and knowing the equilibrium constant, $K$ (or better $\Delta S^{0}$ and $\Delta H^{0}$ ), the dependences on temperature, $p_{\mathrm{H}_{2} \mathrm{O}}$, and acceptor concentration, $[\mathrm{A} /]$, can be calculated. Further, concentrations of other defects can be calculated from the electroneutrality condition or from equilibria with protons. Rearranging Eq. (1.7), we have:

$$
\left[\mathrm{v}_{\mathrm{O}}^{\bullet \bullet}\right]=\frac{[\mathrm{A} /]-\left[\mathrm{OH}_{\mathrm{O}}^{\bullet}\right]}{2}
$$

where $\left[\mathrm{OH}_{\mathrm{O}}^{\bullet}\right]$ is inserted from Eq. (1.8). The concentration of defect electrons is in turn obtained from their equilibrium with oxygen vacancies, or from their equilibrium (Eq. 1.4) with hydrogen above, to become 


$$
n=\frac{K_{2 \mathrm{H} 2 \mathrm{e}}^{1 / 2} p_{\mathrm{H}_{2}}^{1 / 2}[\mathrm{O}]}{\left[\mathrm{OH}_{\mathrm{O}}^{\bullet}\right]}=\frac{K_{2 \mathrm{H} 2 \mathrm{e}}^{1 / 2} p_{\mathrm{H}_{2} \mathrm{O}}^{1 / 2}[\mathrm{O}]}{K_{\mathrm{H}_{2} \mathrm{O}}^{1 / 2} p_{\mathrm{O}_{2}}^{1 / 4}\left[\mathrm{OH}_{\mathrm{O}}^{\bullet}\right]}
$$

where, again, $\left[\mathrm{OH}_{\mathrm{O}}^{\bullet}\right]$ is inserted from Eq. (1.8). Finally, the concentration of electron holes, $p$, is found from the intrinsic electronic ionization equilibrium as $p=K_{\text {in }} / n$.

In some acceptor-doped materials, electron holes may also become dominating defects at high oxygen activities, so that we may consider a more complicated defect structure and electroneutrality:

$$
2\left[\mathrm{v}_{\mathrm{O}}^{\bullet \bullet}\right]+\left[\mathrm{OH}_{\mathrm{O}}^{\bullet}\right]+p=\left[\mathrm{A}^{\prime}\right]=\text { constant }
$$

A situation with all these three positively charged defects being important in compensating the acceptors may possibly be found in some basic (Ba-containing) perovskites such as $\mathrm{BaPrO}_{3}$ and $\mathrm{BaTbO}_{3}$ with very ambivalent $\mathrm{B}$-site cations. The solution to the defect concentrations becomes complex, but can be brought into analytical form. The lack of practical importance leads us instead to limit ourselves to the more common case in which electron holes alone compensate the acceptors:

$$
p=\left[\mathrm{A}^{/}\right]=\text {constant }
$$

whereby, via Eq. (1.4) and $K_{\mathrm{in}}=n \cdot p$, the minor concentration of protons becomes:

$$
\left[\mathrm{OH}_{\mathrm{O}}^{\bullet}\right]=\frac{K_{2 \mathrm{H} 2 \mathrm{e}}^{1 / 2} p_{\mathrm{H}_{2}}^{1 / 2}[\mathrm{O}][\mathrm{A} /]}{K_{\mathrm{in}}}
$$

This will, for instance, be valid in certain ranges of oxygen activity and temperature for many acceptor-doped $\mathrm{LaMO}_{3}(\mathrm{M}=$ transition metal) perovskites with small band gaps.

\subsection{6}

\section{Diffusivity, Mobility and Conductivity: The Nernst-Einstein Relation}

We have above introduced concentrations and relations between concentrations of species of interest. The area-specific flux (flux density) of a species, i, resulting from a driving force, $\mathrm{F}$, is proportional to its concentration and to its mechanical mobility (ease of movement): $j_{\mathrm{i}}=c_{\mathrm{i}} \cdot B_{\mathrm{i}} \cdot F_{\mathrm{i}}$ as we come back to in the next section. First we briefly recall from textbooks that for species with an activated diffusion, the self-diffusion coefficient, $D$, mechanical mobility, $B$, charge mobility, $u$, and conductivity, $\sigma$, are linked through the Nernst-Einstein relation (1.14):

$$
\sigma_{\mathrm{i}}=z_{\mathrm{i}} F c_{\mathrm{i}} u_{\mathrm{i}}=\left(z_{\mathrm{i}} F\right)^{2} c_{\mathrm{i}} B_{\mathrm{i}}=\frac{\left(z_{\mathrm{i}} F\right)^{2} c_{\mathrm{i}} D_{\mathrm{i}}}{R T}
$$


where $D=D_{0} \exp \frac{-Q_{m}}{R T}$ defines the temperature dependence and activation energy, $Q_{m}$. We here use the Faraday constant, $F=96485 \mathrm{C} \mathrm{mol}^{-1}$, expressing the charge per mole of charges, and the defect concentration, $c_{\mathrm{i}}$, must thus be brought into the form of a molar volume concentration. Thus, from the molar fractions, [i], as we have derived them in this section, one needs to multiply by the molar volume density, $d_{\mathrm{M}}\left(\mathrm{mol} \mathrm{cm}^{-3}\right.$ or $\left.\mathrm{mol} \mathrm{m}^{-3}\right): c_{\mathrm{i}}=[i] d_{\mathrm{M}, \mathrm{i}}$. We note that diffusivity and mobility terms do not contain concentration, while conductivity does.

For species that migrate by other mechanisms than diffusion (notably itinerant electrons and holes), the diffusion coefficient is undefined, and instead one uses mobilities with temperature dependences typical of band transport with phonon-, impurity-, or large polaron-limited transport [8].

\section{4}

\section{Wagner Transport Theory for Dense Ceramic Hydrogen-Separation Membranes}

\subsection{1}

\section{General Expressions}

A force, expressed as the negative (down-hill) gradient in a potential, $P_{\mathrm{i}}$, acting on the species, $i$, gives rise to a net flux density, $j_{\mathrm{i}}$, (in moles per unit area per second) of that species which is proportional to its mechanical mobility, $B_{\mathrm{i}}$. By letting the acting potential be the electrochemical potential, $\eta_{\mathrm{i}}$, we obtain in the one-dimensional case:

$$
j_{\mathrm{i}}=B_{\mathrm{i}} c_{\mathrm{i}} \frac{-\mathrm{d} P_{\mathrm{i}}}{\mathrm{d} x}=B_{\mathrm{i}} c_{\mathrm{i}} \frac{-\mathrm{d} \eta_{\mathrm{i}}}{\mathrm{d} x}=-B_{\mathrm{i}} c_{\mathrm{i}}\left[\frac{\mathrm{d} \mu_{\mathrm{i}}}{\mathrm{d} x}+z_{\mathrm{i}} F \frac{\mathrm{d} \varphi}{\mathrm{d} x}\right]
$$

For charged species we commonly substitute in conductivity from Eq. (1.14) and obtain:

$$
j_{\mathrm{i}}=\frac{-\sigma_{\mathrm{i}}}{\left(z_{\mathrm{i}} F\right)^{2}}\left[\frac{\mathrm{d} \mu_{\mathrm{i}}}{\mathrm{d} x}+z_{\mathrm{i}} F \frac{\mathrm{d} \varphi}{\mathrm{d} x}\right]
$$

The flux density for the species, $i$, gives rise to a partial current density, $i_{\mathrm{i}}$ :

$$
i_{\mathrm{i}}=z_{\mathrm{i}} F j_{\mathrm{i}}=\frac{-\sigma_{\mathrm{i}}}{z_{\mathrm{i}} F}\left[\frac{\mathrm{d} \mu_{\mathrm{i}}}{\mathrm{d} x}+z_{\mathrm{i}} F \frac{\mathrm{d} \varphi}{\mathrm{d} x}\right]
$$

The net current density in the sample is obtained by summing the partial current densities over all the species, $k$, and for a membrane (that does not have electrodes or an external circuit) it is necessarily zero (open circuit):

$$
i_{\text {tot }}=\sum_{k} z_{k} F j_{k}=-\sum_{k} \frac{\sigma_{k}}{z_{k} F}\left[\frac{\mathrm{d} \mu_{k}}{\mathrm{~d} x}+z_{k} F \frac{\mathrm{d} \varphi}{\mathrm{d} x}\right]=0
$$


By using the definitions of total conductivity, $\sigma_{\text {tot }}=\sum_{k} \sigma_{k}$, and transport number, $t_{k}=\frac{\sigma_{k}}{\sigma_{\text {tot }}}=\frac{\sigma_{k}}{\sum_{k} \sigma_{k}}$, we obtain the following important expression for the electrical potential gradient through the sample in terms of the transport number and chemical potential gradient of all charge carriers:

$$
\frac{d \varphi}{\mathrm{d} x}=-\sum_{k} \frac{t_{k}}{z_{k} F} \frac{\mathrm{d} \mu_{k}}{\mathrm{~d} x}
$$

\subsection{2}

\section{From Charged to Well-Defined Species: The Electrochemical Equilibrium}

The chemical potentials of charged species are not well-defined, and we need to represent them instead by chemical potentials of neutral species. For this purpose, we may assume equilibria between neutral and charged species and electrons, in the electrochemical redox reaction:

$$
\mathrm{E}=\mathrm{E}^{z}+z \mathrm{e}^{-}
$$

where $\mathrm{E}$ is a neutral chemical entity and $z$ may be positive or negative. The equilibrium condition for this is expressed in terms of the chemical potentials of products and reactants:

$$
\mathrm{d} \mu_{\mathrm{E}^{2}}+z \mathrm{~d} \mu_{\mathrm{e}^{-}}-\mathrm{d} \mu_{\mathrm{E}}=0 \text { or } \mathrm{d} \mu_{\mathrm{E}^{z}}=\mathrm{d} \mu_{\mathrm{E}}-z \mathrm{~d} \mu_{\mathrm{e}^{-}}
$$

We may now insert this for all ionic species, $n$, in the expression for the electrical potential gradient. The entry (among $k$ ) for electrons is left unsubstituted.

When required, we may substitute the chemical potential of the neutral species with activities and partial pressures. For isothermal conditions and for ideal gases we obtain:

$$
\mathrm{d} \mu_{\mathrm{E}}=\mathrm{RTd} \ln a_{\mathrm{E}}=\mathrm{RTd} \ln p_{\mathrm{E}}
$$

\section{4 .3}

\section{The Voltage Over a Sample}

By inserting for the chemical potentials of ions and by using $\sum_{k} t_{k}=1$ we obtain from Eq. (1.19):

$$
\frac{\mathrm{d} \varphi}{\mathrm{d} x}=-\sum_{n} \frac{t_{n}}{z_{n} F} \frac{\mathrm{d} \mu_{n}}{\mathrm{~d} x}+\frac{1}{F} \frac{d \mu_{\mathrm{e}^{-}}}{\mathrm{d} x}
$$

where $n$ represents all the ionic species and for which the chemical potentials, $\mu_{\mathrm{n}}$, now refer to the neutral form. 
We now integrate the electrical potential gradient over the thickness of the sample, from Side I to Side II, in order to obtain the voltage, $U$, over the sample:

$$
\begin{aligned}
& U_{\mathrm{II}-\mathrm{I}}=\int_{\mathrm{I}}^{\mathrm{II}} \mathrm{d} \varphi=-\sum_{n} \int_{\mathrm{I}}^{\mathrm{II}} \frac{t_{n}}{z_{n} F} \mathrm{~d} \mu_{n}+\int_{\mathrm{I}}^{\mathrm{II}} \frac{1}{F} \mathrm{~d} \mu_{\mathrm{e}^{-}} \\
& U_{\mathrm{II}-\mathrm{I}}=\varphi_{\mathrm{II}}-\varphi_{\mathrm{I}}=-\sum_{n} \int_{\mathrm{I}}^{\mathrm{II}} \frac{t_{n}}{z_{n} F} \mathrm{~d} \mu_{n}+\frac{1}{F}\left(\mu_{\mathrm{e}^{-}}^{\mathrm{II}}-\mu_{\mathrm{e}^{-}}^{\mathrm{I}}\right)
\end{aligned}
$$

We further assume that the voltage is measured using the same inert metal on both sides, for example, platinum. This eliminates the difference between chemical potentials of electrons on the two sides, and the voltage measured between the two sides is:

$$
U_{\mathrm{II}-\mathrm{I}}=-\sum_{n} \int_{\mathrm{I}}^{\mathrm{II}} \frac{t_{n}}{z_{n} F} \mathrm{~d} \mu_{n}
$$

This is the basis for establishing transport numbers based on open circuit voltage measurements of cells exposed to a well-defined gradient in chemical activities. If one manages to establish a gradient in the chemical potential of only one species at a time, the transport number of the ion of that species can be determined. If the gradient is small, then the transport numbers may be taken to be constant and equal to an average value, so as to simplify the integration.

\subsection{4}

\section{Flux of a Particular Species}

One of the general expressions for the electrical potential gradient can now be inserted into the expression for the flux density of a particular species (Eq. (1.15)) or the corresponding partial current density (Eq. (1.17)). Since these two sum over chemical potential gradients of charged species, we may conveniently use Eq. (1.19) for our purpose. Inserting this into Eq. (1.15) and rearranging yields:

$$
j_{\mathrm{i}}=\frac{-\sigma_{\mathrm{i}}}{\left(z_{\mathrm{i}} F\right)^{2}}\left[\frac{\mathrm{d} \mu_{\mathrm{i}}}{\mathrm{d} x}-z_{\mathrm{i}} \sum_{k} \frac{t_{k}}{z_{k}} \frac{\mathrm{d} \mu_{k}}{\mathrm{~d} x}\right]
$$

This is a rather general expression, which we can use to calculate flux densities of one charged species in the company of many other species. However, it reflects the flux density and gradients and properties at a particular point in the membrane. The gradients will adjust according to the varying materials properties so as to maintain a constant flux density everywhere - what we call steady state. In 
order to implement this, we integrate the flux density expression over the thickness of the membrane and require that the flux density remains constant:

$$
\int_{\text {I }}^{\text {II }} j_{\mathrm{i}} \mathrm{d} x=j_{\mathrm{i}} L=-\int_{\mathrm{I}}^{\mathrm{II}} \frac{\sigma_{\mathrm{i}}}{\left(z_{\mathrm{i}} F\right)^{2}}\left[\mathrm{~d} \mu_{\mathrm{i}}-z_{\mathrm{i}} \sum_{k} \frac{t_{k}}{z_{k}} \mathrm{~d} \mu_{k}\right]
$$

or

$$
j_{\mathrm{i}}=\frac{-1}{\left(z_{\mathrm{i}} F\right)^{2} L} \int_{\mathrm{I}}^{\mathrm{II}} \sigma_{\mathrm{i}}\left[\mathrm{d} \mu_{\mathrm{i}}-z_{\mathrm{i}} \sum_{k} \frac{t_{k}}{z_{k}} \mathrm{~d} \mu_{k}\right]
$$

where $L$ is the thickness of the membrane and $\mathrm{k}$ now represents all charge carriers. Again, we may substitute the chemical potentials of all ions with that of the neutral species, so that in the following expression, $n$ represents all the ions considered in the total transport system, $\mu_{n}$ and $\mu_{n=i}$ are the chemical potentials of the corresponding neutral species:

$$
j_{\mathrm{i}}=\frac{-1}{\left(z_{\mathrm{i}} F\right)^{2} L} \int_{\mathrm{I}}^{\mathrm{II}} \sigma_{\mathrm{i}}\left[\mathrm{d} \mu_{n=i}-z_{\mathrm{i}} \sum_{n} \frac{t_{n}}{z_{n}} \mathrm{~d} \mu_{n}\right]
$$

\section{4 .5}

\section{Fluxes in a Mixed Proton, Oxygen Ion, and Electron Conductor}

We can now write the flux of protons (species i) through an oxide with mixed conductivity of protons, oxygen ions and electrons (species $k$ ). In this case, the neutral species $(n)$ are hydrogen and oxygen, and we obtain:

$$
j_{\mathrm{H}^{+}}=\frac{-1}{F^{2} L} \int_{\mathrm{I}}^{\mathrm{II}} \sigma_{\mathrm{H}^{+}}\left[\left(t_{\mathrm{O}^{2-}}+t_{\mathrm{e}^{-}}\right) \mathrm{d} \mu_{\mathrm{H}}+\frac{1}{2} t_{\mathrm{O}^{2-}} \mathrm{d} \mu_{\mathrm{O}}\right]
$$

We substitute to obtain an expression containing the partial pressures of the molecular neutral species:

$$
j_{\mathrm{H}^{+}}=\frac{-R T}{4 F^{2} L} \int_{\mathrm{I}}^{\mathrm{II}} \sigma_{\mathrm{H}^{+}}\left[2\left(t_{\mathrm{O}^{2-}}+t_{\mathrm{e}^{-}}\right) \mathrm{d} \ln p_{\mathrm{H}_{2}(\mathrm{~g})}+t_{\mathrm{O}^{2-}} \mathrm{d} \ln p_{\mathrm{O}_{2}(\mathrm{~g})}\right]
$$

From this equation, we see that there are three sources of flux of protons, all proportional to the proton conductivity. A gradient in hydrogen activity drives the protons, and we get a flux if there is a compensating current from fluxes in oxygen ions or electrons. Similarly, a gradient in oxygen activity drives oxygen ions if they 
have sufficient transport number and gives a compensating current of protons. In both cases in which oxygen ions are involved, the hydrogen comes through as water, whereas with electrons the hydrogen comes through as elemental hydrogen.

It is often practical to insert the equilibrium between hydrogen, oxygen and water in order to express the flux density of protons in terms of the water vapor activity gradient:

$$
j_{\mathrm{H}^{+}}=\frac{-R T}{2 F^{2} L} \int_{\mathrm{I}}^{\mathrm{II}} \sigma_{\mathrm{H}^{+}}\left[t_{\mathrm{e}^{-}} \mathrm{d} \ln p_{\mathrm{H}_{2}(\mathrm{~g})}+t_{\mathrm{O}^{2-}} \mathrm{d} \ln p_{\mathrm{H}_{2} \mathrm{O}(\mathrm{g})}\right]
$$

This more clearly shows how the proton flux comes about as fluxes of hydrogen and water.

We may also similarly derive the flux density of oxygen ions:

$$
j_{\mathrm{O}^{2-}}=\frac{-R T}{8 F^{2} L} \int_{\mathrm{I}}^{\mathrm{II}} \sigma_{\mathrm{O}^{2-}}\left[\left(t_{\mathrm{e}^{-}}+t_{\mathrm{H}^{+}}\right) \mathrm{d} \ln p_{\mathrm{O}_{2}(\mathrm{~g})}+2 t_{\mathrm{H}^{+}} \mathrm{d} \ln p_{\mathrm{H}_{2}(\mathrm{~g})}\right]
$$

It has contributions from ambipolar transport of oxygen ions and electrons in an oxygen activity gradient, which corresponds to flux of oxygen through a mixedconducting membrane without proton transport, and from ambipolar transport of oxygen and protons in an oxygen or hydrogen gradient, that is, transport of water. Rewritten in terms of water vapor pressure it becomes:

$$
j_{\mathrm{O}^{2-}}=\frac{R T}{8 F^{2} L} \int_{\mathrm{I}}^{\mathrm{II}} \sigma_{\mathrm{O}^{2-}}\left[t_{\mathrm{e}^{-}} \mathrm{d} \ln p_{\mathrm{O}_{2}(\mathrm{~g})}+2 t_{\mathrm{H}^{+}} \mathrm{d} \ln p_{\mathrm{H}_{2} \mathrm{O}(\mathrm{g})}\right]
$$

\subsection{6}

\section{Fluxes in a Mixed Proton and Electron Conductor}

By assuming that the transport number for oxygen ions is zero (cf. Eq. (1.32)), the only flux of hydrogen remaining is that of ambipolar transport of protons and electrons:

$$
j_{\mathrm{H}^{+}}=\frac{-R T}{2 F^{2} L} \int_{\mathrm{I}}^{\mathrm{II}} \sigma_{\mathrm{H}^{+}} t_{\mathrm{e}^{-}} \mathrm{d} \ln p_{\mathrm{H}_{2}(\mathrm{~g})}
$$

This is a good point at which to look a little closer at the ambipolar transport term that we have mentioned earlier in passing. The materials property of interest here is the protonic-electronic ambipolar conductivity term, which can be written in 
many ways: $\sigma_{\mathrm{H}^{+}} t_{\mathrm{e}^{-}}=\sigma_{\text {total }} t_{\mathrm{H}^{+}} t_{\mathrm{e}^{-}}=\sigma_{\mathrm{e}^{-}} t_{\mathrm{H}^{+}}=\frac{\sigma_{\mathrm{H}^{+}} \sigma_{\mathrm{e}^{-}}}{\sigma_{\text {total }}}$. It may be noted that if - and only if - the material conducts no other species than protons and electrons can it also be expressed in the commonly used form $\frac{\sigma_{\mathrm{H}^{+}} \sigma_{\mathrm{e}^{-}}}{\sigma_{\mathrm{H}^{+}}+\sigma_{\mathrm{e}^{-}}}$.

The hydrogen partial pressure gradient is the driving force of the flux in Eq. (1.36). In order to integrate the equation, we need to know how the proton conductivity and electron transport number vary with the hydrogen activity. If we further assume that the material exhibits dominating electronic conduction, then the electronic transport number is unity; $t_{\mathrm{e}^{-}} \approx 1$. In the following, we describe three limiting cases of this, which allow us to integrate the above equation.

If protons are minority defects, then their concentration and the proton conductivity are proportional to $p_{\mathrm{H}_{2}}^{1 / 2}$, and we obtain:

$$
j_{\mathrm{H}^{+}}=\frac{-R T \sigma_{\mathrm{H}^{+}, 0}}{2 F^{2} L} \int_{\mathrm{I}}^{\mathrm{II}} p_{\mathrm{H}_{2}(\mathrm{~g})}^{1 / 2} \mathrm{~d} \ln p_{\mathrm{H}_{2}(\mathrm{~g})}=\frac{-R T \sigma_{\mathrm{H}^{+}, 0}}{2 F^{2} L} \int_{\mathrm{I}}^{\mathrm{II}} p_{\mathrm{H}_{2}(\mathrm{~g})}^{-1 / 2} \mathrm{~d} p_{\mathrm{H}_{2}(\mathrm{~g})}
$$

where $\sigma_{\mathrm{H}^{+}, 0}$ is the proton conductivity at standard hydrogen pressure $\left(10^{5} \mathrm{~Pa} \approx 1 \mathrm{~atm} \mathrm{H}_{2}(\mathrm{~g})\right)$. The integration yields:

$$
j_{\mathrm{H}^{+}}=\frac{-R T \sigma_{\mathrm{H}^{+}, 0}}{F^{2} L}\left[\left(p_{\mathrm{H}_{2}(\mathrm{~g})}^{1 / 2}\right)^{\mathrm{II}}-\left(p_{\mathrm{H}_{2}(\mathrm{~g})}^{1 / 2}\right)^{\mathrm{I}}\right]
$$

If protons are majority defects and compensated by electrons, then their concentration and the proton conductivity are proportional to $p_{\mathrm{H}_{2}}^{1 / 4}$, and we obtain:

$$
j_{\mathrm{H}^{+}}=\frac{-R T \sigma_{\mathrm{H}^{+}, 0}}{2 F^{2} L} \int_{\mathrm{I}}^{\mathrm{II}} p_{\mathrm{H}_{2}(\mathrm{~g})}^{1 / 4} \mathrm{~d} \ln p_{\mathrm{H}_{2}(\mathrm{~g})}=\frac{-R T \sigma_{\mathrm{H}^{+}, 0}}{2 F^{2} L} \int_{\mathrm{I}}^{\mathrm{II}} p_{\mathrm{H}_{2}(\mathrm{~g})}^{-3 / 4} \mathrm{~d} p_{\mathrm{H}_{2}(\mathrm{~g})}
$$

where $\sigma_{\mathrm{H}^{+}, 0}$ is the proton conductivity at standard hydrogen pressure $\left(10^{5} \mathrm{~Pa} \approx\right.$ $\left.1 \mathrm{~atm} \mathrm{H}_{2}(\mathrm{~g})\right)$. The integration yields:

$$
j_{\mathrm{H}^{+}}=\frac{-2 R T \sigma_{\mathrm{H}^{+}, 0}}{F^{2} L}\left[\left(p_{\mathrm{H}_{2}(\mathrm{~g})}^{1 / 4}\right)^{\mathrm{II}}-\left(p_{\mathrm{H}_{2}(\mathrm{~g})}^{1 / 4}\right)^{\mathrm{I}}\right]
$$

If protons are majority defects and compensated by acceptor-dopants, then their concentration and the proton conductivity are independent of $p_{\mathrm{H}_{2}}$, and we obtain:

$$
j_{\mathrm{H}^{+}}=\frac{-R T \sigma_{\mathrm{H}^{+}}}{2 F^{2} L} \int_{\mathrm{I}}^{\mathrm{II}} \mathrm{d} \ln p_{\mathrm{H}_{2}(\mathrm{~g})}=\frac{-R T \sigma_{\mathrm{H}^{+}}}{2 F^{2} L} \int_{\mathrm{I}}^{\mathrm{II}} \frac{1}{p_{\mathrm{H}_{2}(\mathrm{~g})}} \mathrm{d} p_{\mathrm{H}_{2}(\mathrm{~g})}
$$

where $\sigma_{\mathrm{H}^{+}}$is the proton conductivity. The integration yields: 


$$
\begin{aligned}
j_{\mathrm{H}^{+}} & =\frac{-R T \sigma_{\mathrm{H}^{+}}}{2 F^{2} L}\left[\ln p_{\mathrm{H}_{2}(\mathrm{~g})}^{\mathrm{II}}-\ln p_{\mathrm{H}_{2}(\mathrm{~g})}^{\mathrm{I}}\right] \\
& =\frac{-2.303 R T \sigma_{\mathrm{H}^{+}}}{2 F^{2} L}\left[\log p_{\mathrm{H}_{2}(\mathrm{~g})}^{\mathrm{II}}-\log p_{\mathrm{H}_{2}(\mathrm{~g})}^{\mathrm{I}}\right]
\end{aligned}
$$

In the first two cases (protons in minority or compensated by electrons), the hydrogen partial pressure on the high pressure side has a large effect on the flux, while how small the pressure is on the other side has less influence. In the last of the three cases, however, the differences in the logarithms (representing the logarithm of the ratio of the two hydrogen pressures) makes the small and the large pressure become equally important.

The minus sign in front of the flux equations arises from the flux going downhill, that is, in the direction of negative gradient. The flux is here defined as going in the direction from Side I towards Side II and thus becomes positive if the partial pressure of hydrogen is larger at Side I than at Side II.

In the above cases, we assumed that the transport number of electronic defects was unity. Especially in the case for which protons dominate and compensate acceptors, the electronic defects may be sufficiently minor, in terms of concentration, that they do not dominate the conductivity. If we consider as an alternative approximation that the transport number of protons is unity, then we may write:

$$
\begin{aligned}
j_{\mathrm{H}^{+}} & =\frac{-R T}{2 F^{2} L} \int_{\mathrm{I}}^{\mathrm{II}} \sigma_{\mathrm{H}^{+}} t_{\mathrm{e}^{-}} \mathrm{d} \ln p_{\mathrm{H}_{2}(\mathrm{~g})}=\frac{-R T}{2 F^{2} L} \int_{\mathrm{I}}^{\mathrm{II}} \sigma_{\mathrm{e}^{-}} t_{\mathrm{H}^{+}} \mathrm{d} \ln p_{\mathrm{H}_{2}(\mathrm{~g})} \\
& =\frac{-R T}{2 F^{2} L} \int_{\mathrm{I}}^{\mathrm{II}} \sigma_{\mathrm{e}^{-}} \mathrm{d} \ln p_{\mathrm{H}_{2}(\mathrm{~g})}
\end{aligned}
$$

If protons compensate acceptors, then the electronic conductivity is proportional to $p_{\mathrm{H}_{2}}^{1 / 2}$ and $p_{\mathrm{H}_{2}}^{-1 / 2}$, respectively, in the case of electrons (n-type) and holes (p-type) if $p_{\mathrm{O}_{2}}$ is assumed constant. This gives:

$$
\begin{aligned}
j_{\mathrm{H}^{+}} & =\frac{-R T \sigma_{\mathrm{n}, 0}}{2 F^{2} L} \int_{\mathrm{I}}^{\mathrm{II}} p_{\mathrm{H}_{2}}^{1 / 2} \mathrm{~d} \ln p_{\mathrm{H}_{2}(\mathrm{~g})}=\frac{-R T \sigma_{\mathrm{n}, 0}}{2 F^{2} L} \int_{\mathrm{I}}^{\mathrm{II}} p_{\mathrm{H}_{2}}^{-1 / 2} \mathrm{~d} p_{\mathrm{H}_{2}(\mathrm{~g})} \\
& =\frac{-R T \sigma_{\mathrm{n}, 0}}{F^{2} L}\left[\left(p_{\mathrm{H}_{2}}^{1 / 2}\right)^{\mathrm{II}}-\left(p_{\mathrm{H}_{2}}^{1 / 2}\right)^{\mathrm{I}}\right]
\end{aligned}
$$

for limiting n-type conductivity, and 


$$
\begin{aligned}
j_{\mathrm{H}^{+}} & =\frac{-R T \sigma_{\mathrm{p}, 0}}{2 F^{2} L} \int_{\mathrm{I}}^{\mathrm{II}} p_{\mathrm{H}_{2}}^{-1 / 2} \mathrm{~d} \ln p_{\mathrm{H}_{2}(\mathrm{~g})}=\frac{-R T \sigma_{\mathrm{p}, 0}}{2 F^{2} L} \int_{\mathrm{I}}^{\mathrm{II}} p_{\mathrm{H}_{2}}^{-3 / 2} \mathrm{~d} p_{\mathrm{H}_{2}(\mathrm{~g})} \\
& =\frac{R T \sigma_{\mathrm{p}, 0}}{F^{2} L}\left[\left(p_{\mathrm{H}_{2}}^{-1 / 2}\right)^{\mathrm{II}}-\left(p_{\mathrm{H}_{2}}^{-1 / 2}\right)^{\mathrm{I}}\right]
\end{aligned}
$$

for limiting p-type conductivity, where $\sigma_{\mathrm{n}}$ and $\sigma_{\mathrm{p}}$ are electron and hole conductivities, and $\sigma_{\mathrm{n}, 0}$ and $\sigma_{\mathrm{p}, 0}$ are conductivities at standard hydrogen pressure $\left(10^{5} \mathrm{~Pa} \approx\right.$ 1 atm $\left.\mathrm{H}_{2}(\mathrm{~g})\right)$.

There are, of course, other limiting situations that may be considered, for example if protons are compensated by negative point defects such as metal vacancies or oxygen interstitials. These are, however, mainly dominant under oxidizing conditions, not commonly considered for hydrogen-separation processes.

Before we move on to more special cases, we take this opportunity to remind the reader of the many different pressure dependences we get for fluxes of dense membranes, and mixed conducting ones in particular. This is why permeabilities in dense mixed conducting membranes most often cannot be given as simply and in the same units as permeability in other types of membranes. We will return to the consequences of this when we quote example literature values in Section 1.9.1.

\subsection{7}

\section{Fluxes in a Mixed Proton and Oxygen Ion Conductor}

If the material is a mixed proton and oxygen ion conductor and has a negligible transport number for electronic defects, then we have:

$$
j_{\mathrm{H}^{+}}=\frac{-R T}{2 F^{2} L} \int_{\mathrm{I}}^{\mathrm{II}} \sigma_{\mathrm{H}^{+}} t_{\mathrm{O}^{2}} \mathrm{~d} \ln p_{\mathrm{H}_{2} \mathrm{O}(\mathrm{g})}
$$

that is, the water vapor pressure gradient is the driving force. Typically, the oxide is acceptor-doped, and protons and oxygen vacancies compete to be the compensating defect. If oxygen vacancies are dominant, then their concentration is independent of $p_{\mathrm{H}_{2} \mathrm{O}}$, while the proton concentration increases with $p_{\mathrm{H}_{2} \mathrm{O}}^{1 / 2}$. If on the other hand, the protons are dominant and therefore constant, then the concentration of oxygen vacancies decreases with $p_{\mathrm{H}_{2} \mathrm{O}}^{-1}$. Depending on whether the material has a transport number of any of the two that can be set equal to unity, these dependences can be inserted into the remaining limiting conductivity, and the equation easily integrated. 


\subsection{8}

\section{Fluxes in a Mixed Proton, Oxygen Ion, and Electron Conductor Revisited}

In ambipolar diffusion of protons and oxygen ions, the two types of ions move in the same direction for charge compensation, and the net result is permeation of water. If we also have electronic transport, then the transport of hydrogen and oxygen ions may be de-coupled. They may move in the same direction or in opposite directions and at different ratios.

Consider, for instance, a mixed-conducting membrane exposed to wet hydrogen on one side and wet argon on the other. The intention might be to measure the hydrogen permeability by measuring the amount of hydrogen in the argon side permeate gas. If the water vapor pressures are equal, there will be no ambipolar transport of water in any direction. Hydrogen will permeate from the high hydrogen partial pressure side to the low. The flux will be proportional to the ambipolar proton-electron conductivity, and hydrogen will appear in the permeate. But at the same time, oxygen ions will move in the opposite direction, from the higher oxygen activity in the wet argon to the lower in the wet hydrogen, and the oxygen is taken from the water vapor in the argon. This flux is limited by the ambipolar oxygen ion-electron conductivity. As a result of this flux, hydrogen is formed in the argon, and it may be difficult experimentally to distinguish whether the hydrogen arises from proton transport or oxygen ion transport. Only analysis of the water vapor balance on one or both sides - usually difficult enough in itself - can distinguish one from the other or give a correct balance between proton and oxygen ion conductivity. The two opposite fluxes of protons and oxygen ions both give charge transport in the same direction, and they are compensated by a flux of electrons being the sum of the two.

In the case discussed here, where three or more species (e.g., A, B, C) provide charge transport, it may, as mentioned earlier, be important to keep the pairwise ambipolar conductivities in the correct form, and to be aware of the inequality:

$$
\sigma_{A} t_{B}=\sigma_{\text {total }} t_{A} t_{B}=\frac{\sigma_{A} \sigma_{B}}{\sigma_{\text {total }}} \neq \frac{\sigma_{A} \sigma_{B}}{\sigma_{A}+\sigma_{B}} .
$$

\section{4 .9}

\section{Permeation of Neutral Hydrogen Species}

To express the diffusion of hydrogen as neutral species, we can use the same starting point as for charged species: From Eq. (1.15) and by substitution with Eq. (1.14), flux densities of hydrogen atoms and hydrogen molecules expressed in terms of diffusivities become:

$$
j_{\mathrm{H}}=\frac{-D_{\mathrm{H}} c_{\mathrm{H}}}{R T} \frac{\mathrm{d} \mu_{\mathrm{H}}}{\mathrm{d} x}=\frac{-D_{\mathrm{H}} c_{\mathrm{H}}}{2} \frac{\mathrm{d} \ln p_{\mathrm{H}_{2}(\mathrm{~g})}}{\mathrm{d} x}
$$




$$
j_{\mathrm{H}_{2}}=\frac{-D_{\mathrm{H}_{2}} c_{\mathrm{H}_{2}}}{R T} \frac{\mathrm{d} \mu_{\mathrm{H}_{2}}}{\mathrm{~d} x}=-D_{\mathrm{H}_{2}} c_{\mathrm{H}_{2}} \frac{\mathrm{d} \ln p_{\mathrm{H}_{2}(\mathrm{~g})}}{\mathrm{d} x}
$$

Integration over the membrane thickness yields:

$$
\begin{aligned}
& j_{\mathrm{H}}=\frac{-1}{2 L} \int_{\mathrm{I}}^{\mathrm{II}} D_{\mathrm{H}} c_{\mathrm{H}} \mathrm{d} \ln p_{\mathrm{H}_{2}(\mathrm{~g})} \\
& j_{\mathrm{H}_{2}}=\frac{-1}{L} \int_{\mathrm{I}}^{\mathrm{II}} D_{\mathrm{H}_{2}} c_{\mathrm{H}_{2}} \mathrm{~d} \ln p_{\mathrm{H}_{2}(\mathrm{~g})}
\end{aligned}
$$

Assuming that the diffusivities are independent of concentration, and that the concentrations are given by the equilibria with hydrogen partial pressure treated earlier, we obtain:

$$
\begin{aligned}
j_{\mathrm{H}} & =\frac{-D_{\mathrm{H}} K_{2 \mathrm{H}}^{1 / 2}}{2 L} \int_{\mathrm{I}}^{\mathrm{II}} p_{\mathrm{H}_{2}(\mathrm{~g})}^{1 / 2} \mathrm{~d} \ln p_{\mathrm{H}_{2}(\mathrm{~g})}=\frac{-D_{\mathrm{H}} K_{2 \mathrm{H}}^{1 / 2}}{2 L} \int_{\mathrm{I}}^{\mathrm{II}} p_{\mathrm{H}_{2}(\mathrm{~g})}^{-1 / 2} \mathrm{~d} p_{\mathrm{H}_{2}(\mathrm{~g})} \\
& =\frac{-D_{\mathrm{H}} K_{2 \mathrm{H}}^{1 / 2}}{L}\left[\left(p_{\mathrm{H}_{2}(\mathrm{~g})}^{1 / 2}\right)^{\mathrm{II}}-\left(p_{\mathrm{H}_{2}(\mathrm{~g})}^{1 / 2}\right)^{\mathrm{I}}\right] \\
j_{\mathrm{H}_{2}} & =\frac{-D_{\mathrm{H}_{2}} K_{\mathrm{H}_{2}}}{L} \int_{\mathrm{I}}^{\mathrm{II}} p_{\mathrm{H}_{2}(\mathrm{~g})} \mathrm{d} \ln p_{\mathrm{H}_{2}(\mathrm{~g})}=\frac{-D_{\mathrm{H}_{2}} K_{\mathrm{H}_{2}}}{L} \int_{\mathrm{I}}^{\mathrm{II}} \mathrm{d} p_{\mathrm{H}_{2}(\mathrm{~g})} \\
& =\frac{-D_{\mathrm{H}_{2}} K_{\mathrm{H}_{2}}}{L}\left[p_{\mathrm{H}_{2}(\mathrm{~g})}^{\mathrm{II}}-p_{\mathrm{H}_{2}(\mathrm{~g})}^{\mathrm{I}}\right]
\end{aligned}
$$

in which $K_{2 \mathrm{H}}$ and $K_{\mathrm{H} 2}$ are the equilibrium constants of the dissolution of two atoms or one molecule, respectively, from one hydrogen molecule. It may be noted as before that $K_{2 \mathrm{H}}$ is equal to the square of the Sieverts' law constant: $K_{2 \mathrm{H}}=K_{\mathrm{S}}^{2}$.

We note that the molecular flux is proportional to the difference in hydrogen pressure (as in gas phase transport through a porous membrane), whereas atomic transport has a square root dependence, and as such would behave similarly to ambipolar transport of protons and electrons limited by the conductivity of a minority concentration of protons. 
1.4.10

\section{What About Hydride Ions?}

There have been some speculations about the existence and transport of hydride ions $\left(\mathrm{H}^{-}\right)$in oxides under reducing conditions, but according to thermodynamic data of hydrides, the conditions for a hydrogen-separation membrane will be much too oxidizing for hydride ions to be stable [9]. Moreover, the apparent indications of hydride ions in the literature have now been rationalised by other, more credible phenomena, actually arising from transport of neutral hydrogen [2]. Therefore, it seems, at present, that hydride ions play no role in hydrogen permeation in oxides.

\section{5}

\section{Surface Kinetics of Hydrogen Permeation in Mixed Proton-Electron Conductors}

As the mixed proton and electron conductive oxide membrane becomes sufficiently thin, surface kinetics will become important, and diffusion of protons across the membrane will no longer be rate determining for the overall hydrogen flux. Bouwmeester et al. [10] defined a characteristic thickness, $L_{C}$, for membranes where surface kinetics and bulk kinetics are equally important to the flux. Decreasing a membrane's thickness below $L_{C}$ gives essentially no increase in the flux.

To the best of our knowledge, there is yet no literature example where surface kinetics has been proven to limit the hydrogen permeation across a mixed proton-electron conducting oxide, and seemingly membrane thicknesses below $L_{C}$ have thus never been reached.

Generally, one may divide the overall surface reaction into adsorption-dissociation of the reacting gas molecule, and incorporation of the dissociated species. Again these may consist of more elementary reaction sequences (adsorption, dissociation, surface diffusion, charge transfer, several incorporation steps). Hence, the overall series of reactions describing the process from a gas molecule to a protonic defect in the oxide may be complex. However, one step is often much slower than the others and is defined as the rate-determining step (RDS). One may envisage different mechanisms involved in the surface reaction for different hydrogen-containing species, for example, $\mathrm{H}_{2}, \mathrm{H}_{2} \mathrm{O}, \mathrm{NH}_{3}, \mathrm{H}_{2} \mathrm{~S}$ and different hydrocarbons. However, in the treatment below we will focus on the reaction between hydrogen, $\mathrm{H}_{2}$, and the oxide surface.

Rates of hydrogen dissociation on mixed proton-electron conductors have, up to now, hardly been studied. Dissociation of $\mathrm{H}_{2} / \mathrm{D}_{2}$ on metallic surfaces has, on the other hand, been extensively investigated as a measure for hydrogen activation. In the simplest mechanism, hydrogen is dissociatively adsorbed on the metallic surface forming two independent hydrogen atoms. Recombination and desorption accordingly result in exchange of partner atoms. It has also been suggested that exchange may occur via triatomic intermediates. These two mecha- 
nisms are usually referred to as the Bonhoeffer-Farkas and the Rideal-Eley mechanisms $[11,12]$. The former has been observed to be operative usually at higher temperatures whereas the latter, involving intermediates, is operative only at low temperatures.

In the case of oxides, the catalytic activity towards $\mathrm{H}_{2}$ dissociation has been investigated for several binary oxides including $\mathrm{Cr}_{2} \mathrm{O}_{3}, \mathrm{Co}_{3} \mathrm{O}_{4}, \mathrm{NiO}, \mathrm{ZnO}, \mathrm{Al}_{2} \mathrm{O}_{3}$, and some of the rare earth sesquioxides [13-16]. Most of these studies have been performed below room temperature, and are, as such, not directly relevant for the high temperature mixed proton-electron conductors. One may conclude, however, from these experimental results that for higher temperatures, hydrogen dissociation occurs through dissociative adsorption and desorption following Bonhoeffer-Farkas type mechanisms. On this basis, hydrogen dissociation can be treated according to the Langmuir equation. The simplest route from hydrogen gas to protons dissolved in the oxide may be written:

$$
\begin{aligned}
& \mathrm{H}_{2}(\mathrm{~g})+2 \mathrm{v}_{\mathrm{ads}}=2 \mathrm{H}_{\mathrm{ads}} \\
& \mathrm{H}_{\mathrm{ads}}+\mathrm{O}_{\mathrm{O}}^{\mathrm{X}}=\mathrm{OH}_{\mathrm{O}}^{\bullet}+\mathrm{e}^{\prime}+\mathrm{v}_{\mathrm{ads}}
\end{aligned}
$$

As an alternative to forming electrons, hydrogen may be oxidized by annihilation of electron holes.

These reactions may be divided again into different possible intermediate steps, for example, including charged adsorbed hydrogen species (e.g., $\mathrm{H}_{2}^{+}$). One should recognize the difference between the redox reaction in Eqs. (1.53) and (1.54) and reactions where water is the source of proton incorporation as an acid/base reaction.

Surface defects are likely to act as catalytically active centers at which hydrogen dissociation takes place. The electronic states of these defects may be located within the bandgap of the semiconducting oxide and are likely to be involved in the electron transfer between hydrogen and the membrane [17]. In the reaction in Eq. (1.53), we have not given any indication as to which sites are active for adsorption. However, many of these materials are acceptor doped, and one may expect the dopants or their compensating defects to be involved. One might speculate, for instance, whether oxygen vacancies act as electron sinks as an intermediate step, here shown with two-electron steps:

$$
\begin{aligned}
& \mathrm{H}_{2}(\mathrm{~g})+\mathrm{v}_{\mathrm{O}}^{\bullet \bullet}+2 \mathrm{O}_{\mathrm{O}}^{\mathrm{x}}=2 \mathrm{OH}_{\mathrm{O}}^{\bullet}+\mathrm{v}_{\mathrm{O}}^{\mathrm{x}} \\
& \mathrm{v}_{\mathrm{O}}^{\mathrm{x}}=\mathrm{v}_{\mathrm{O}}^{\bullet \bullet}+2 \mathrm{e}^{\prime}
\end{aligned}
$$

The reaction stages and species involved may be expected to be similar on the feed and permeate sides of a hydrogen membrane. If the flux is driven by reaction with oxygen on the permeate side, this side may naturally be quite different, both in terms of ionic species and electronic (holes versus electrons). The formation or presence of water vapor may also play a significant role in hydrogen exchange kinetics. 
When surface reaction limitations are in control, there is a discontinuity or a steep gradient in the chemical potential of hydrogen between the gas phase and the initial bulk layer at the surface. This may have the form of a gradient in the gas phase next to the surface or in the actual locus between the gas phase and the surface. Depending on the degree of surface control, more or less of the overall chemical potential gradient in the system will be set in an interfacial layer between the potential in the gas phase $\mu_{\mathrm{H}}(\mathrm{g})$ and the surface $\mu_{\mathrm{H}}$ (surf). The flux of hydrogen across the surface interfacial region may now be expressed in terms of the concentrations of hydrogen at the start and end of the interface $\left(c_{\mathrm{H}}(\mathrm{g})\right.$ and $c_{\mathrm{H}}$ (surf)) and the phenomenological rate constant, $k$, or in terms of the change in concentration, $\mathrm{d} c_{\mathrm{H}}$, at the very rate-determining step and the rate constant, $\Re_{0}$ :

$$
j_{\mathrm{H}}=k\left[c_{\mathrm{H}}(\mathrm{g})-c_{\mathrm{H}}(\text { surf })\right]=-\Re_{0} \mathrm{~d} c_{\mathrm{H}}
$$

By using $\mu_{\mathrm{H}}=\mu_{\mathrm{H}}^{0}+R T \ln a_{\mathrm{H}} \approx \mu_{\mathrm{H}}^{0}+R T \ln c_{\mathrm{H}}$ and $\mathrm{d} \ln c_{\mathrm{H}}=\mathrm{d} c_{\mathrm{H}} / c_{\mathrm{H}}$ we may rewrite the right hand part into:

$$
j_{\mathrm{H}_{2}}=\frac{j_{\mathrm{H}}}{2}=-\Im_{0} \frac{\mathrm{d} \mu_{\mathrm{H}_{2}}^{\text {int }}}{R T}
$$

where $\Im_{0}=\frac{1}{4} c_{\mathrm{H}} \cdot \Re_{0}$ where $c_{\mathrm{H}}$ is now the average concentration of hydrogen at the rate determining step. $\Im_{0}$ is known as the balanced exchange rate and represents the coefficient between driving force (chemical potential step) and flux. This is useful when one needs to relate the chemical potential change over the interface (i.e., over the rate-determining step) with the remaining changes over the bulk of the membrane in order to compare bulk and interface kinetics and find $L_{\mathrm{C}}$.

In order to illustrate the possible further analysis, let us assume that the surface kinetics is rate determined by dissociative adsorption (Eq. (1.53)). The net rate then corresponds to:

$$
r=\vec{k}\left[\mathrm{v}_{\mathrm{ads}}\right]^{2} \cdot p_{\mathrm{H}_{2}}-\bar{k}\left[\mathrm{H}_{\mathrm{ads}}\right]^{2}
$$

Since this treatment is restricted to situations close to equilibrium (linear approximation of the Taylor series [18]), the net rate, $r$, is close to zero and consequently:

$$
\vec{k}\left[\mathrm{v}_{\mathrm{ads}}\right]^{2} \cdot p_{\mathrm{H}_{2}}=\bar{k}\left[\mathrm{H}_{\mathrm{ads}}\right]^{2}
$$

On this basis the rate constant $\Re_{0}$ may be represented by:

$$
\begin{aligned}
\Re_{0} & =\vec{k}\left[\mathrm{v}_{\mathrm{ads}}\right]^{2} \cdot p_{\mathrm{H}_{2}}=\bar{k}\left[\mathrm{H}_{\mathrm{ads}}\right]^{2}=\sqrt{\vec{k} \cdot \grave{k} \cdot p_{\mathrm{H}_{2}}\left[\mathrm{v}_{\mathrm{ads}}\right]^{2} \cdot\left[\mathrm{H}_{\mathrm{ads}}\right]^{2}} \\
& =\left[\mathrm{v}_{\mathrm{ads}}\right] \cdot\left[\mathrm{H}_{\mathrm{ads}}\right] \sqrt{\vec{k} \cdot \grave{k} \cdot p_{\mathrm{H}_{2}}}
\end{aligned}
$$


where concentrations refer to conditions sufficiently close to equilibrium. In our particular example of assuming dissociative adsorption as rate limiting (Eq. (1.53)), we thus arrive at a $p_{\mathrm{H}_{2}}^{1 / 2}\left[\mathrm{v}_{\mathrm{ads}}\right]\left[\mathrm{H}_{\mathrm{ads}}\right]$ dependence for the rate constant $\Re_{0}$. At low coverage of hydrogen, we may, from Eq. (1.53), assume that $\left[\mathrm{v}_{\mathrm{ads}}\right]=$ const. and $\left[\mathrm{H}_{\mathrm{ads}}\right] \propto \mathrm{p}_{\mathrm{H}_{2}}^{1 / 2}$ so that the final dependence is $\Re_{0} \propto p_{\mathrm{H}_{2}}^{1 / 4}$. At high coverage we see in the same manner that $\Re_{0}$ becomes independent of $p_{\mathrm{H}_{2}} \cdot \Re_{0}$ can now be inserted in $\Im_{0}$ and $j_{\mathrm{H}_{2}}$ as above.

\section{6}

\section{Issues Regarding Metal Cation Transport in Hydrogen-permeable Membrane Materials}

In oxygen-permeable membranes operating in oxygen activity gradients, the metal cations in the membrane feel an opposite gradient in their own chemical potential; they are drawn towards the high oxygen (feed) side. This may eventually lead to demixing and in some cases decomposition of the membrane compound. In dense, ceramic hydrogen-separation membranes in which hydrogen is extracted by reaction with oxygen in air, the situation will be much the same.

However, if the process is driven by sweeping with an inert gas or by pumping, the oxygen activity difference may be relatively small, zero, or even reversed. The requirements for small cation diffusivities may, therefore, be eased. One may even stop cation diffusion in such cases completely by adjusting the water vapor contents until the oxygen activity gradient is zero. One may also deliberately employ modest temperature gradients to counteract possible isothermal oxygen activity gradients. Aside from driving forces for cations set up by gradients in their chemical potentials, cations may, in principle, also experience drag from migrating protons, but this is probably not very strong, and examples are not known to us.

\section{7}

\section{Modeling Approaches}

The term modeling covers widely different calculational (computer-aided) methodologies and tools used to handle complex sets of equations and large numbers of iterative calculations. We have chosen to include three such modeling issues in this section, although they may have little in common in aim, methodology, and computer intensiveness: Defect structures, flux systems, and atomic simulations.

We have seen above that two or three defect species may dominate the defect structure simultaneously, and we have derived analytical expressions for their concentrations, which may be used in modeling fluxes and membrane behavior. Different approaches exist for the increased complexity of cases with more defects: Sequential analytical breakdown of the set of equations (equilibria and charge, mass, and site balances) can be carried out by a computer program or spreadsheet 
[19] (and has been applied to analysis of mixed conducting hydrogen permeable membranes [20]). This approach calculates all the concentrations quickly, and is suitable for data fitting. It requires reprogramming for each new defect structure type. In a semi-numerical approach, the electroneutrality condition is solved numerically for one defect (e.g., electrons) after all other defect concentrations are replaced manually by equilibria and mass and site balances relating them to the chosen defect. This approach also converges very fast once the expressions are entered. Finally, a truly numerical approach may read all equations, and then solve them numerically. Because the equations are mixtures of linear and nonlinear equations, and because some numbers are very small, algorithms must be made robust, and are consequently slow. One needs not enter any mathematics just the definitions of the individual equations. This approach may thus quickly solve new systems, but is too slow to allow efficient data fitting.

Gradients and fluxes may vary both across and along membranes. When one adds changing limiting defect and transport situations, the overall modeling of a membrane may become very complex. In the finite element modeling approach, the membrane is split up into small geometrical slices (elements). In three dimensions one uses finite elements; in two dimensions finite areas; and in one dimension finite lengths. Within each element, the gradients and properties are constant (e.g., set as an average within the element) so that transport equations become simple. Concentrations and fluxes must connect at boundaries between elements. Steady state may be imposed by equaling the fluxes in all elements in a particular direction, but transient behaviors may also be modeled.

Finite length modeling is suitable for analyzing transport through a membrane, including the gas phase and surfaces. For analyzing the situation for gases flowing along a membrane, one may use finite area modeling in which each finite area is a piece of the membrane with linear behavior and predictable flux density. For an example of finite element modeling of fluxes in proton-containing materials, see Ref. [21].

Atomistic simulations can nowadays reproduce the structure and elastic and dielectric properties of a range of solids quite well, using various calculational methods, models, and approximations. On the one hand we have static lattice simulations of idealized ion spheres with predetermined radial charge density functions, interatomic potentials, and polarizabilities - allowing large numbers of ions to be included in the cell to be calculated. On the other hand we have quantum mechanical dynamic simulations and ab initio methods, where the accuracy and credibility is higher, but where the number of atoms in the modeled volume must be kept relatively small. Protons turn out to be difficult to handle in any such modeling, since they cannot be represented by classical spheres like other ions. Still, various methods yield qualitative predictions of protons' thermodynamics in oxides [22]. Quantum mechanical treatment of the valence electrons has enabled modeling of migration of protons on a detailed level locally, and has provided our present understanding of proton rotation, and jumping, and the effect of the host lattice dynamics [23]. 
1.8

\section{Experimental Techniques and Challenges}

\subsection{1}

\section{Investigation of Fundamental Materials Properties}

\subsubsection{Concentration}

In investigations of proton conducting and mixed proton conducting oxides, one is usually interested in finding the concentration of protons and their role in the defect structure. Absolute measures of the proton content can be determined with a number of methods, most commonly and simply by thermogravimetry (TG). Various procedures are in use:

One may measure the weight of a sample in a certain atmosphere versus temperature (isobars). This can be done in an oxidizing atmosphere $\left(\mathrm{O}_{2}+\mathrm{H}_{2} \mathrm{O}+\right.$ inert; $p_{\mathrm{O}_{2}}, p_{\mathrm{H}_{2} \mathrm{O}}=$ constant $)$ or in a reducing atmosphere $\left(\mathrm{H}_{2}+\mathrm{H}_{2} \mathrm{O}+\right.$ inert; $p_{\mathrm{H}_{2}}$, $p_{\mathrm{H}_{2} \mathrm{O}}=$ constant). One may alternatively measure the weight at fixed temperature (isotherm), for example, versus $p_{\mathrm{H}_{2}}$ or $p_{\mathrm{H}_{2} \mathrm{O}}$. Parameters of the defect reaction and, in turn, proton concentration versus temperature and partial pressures are extracted by modeling the weight change using the appropriate defect structure, for example, using Eq. (1.5) for protons compensated by electrons (weight change reflects $\mathrm{H}_{2}$ ) or Eq. (1.8) for acceptor-doped oxides dominated by oxygen vacancies and protons (weight change reflects $\mathrm{H}_{2} \mathrm{O}$ ). See Refs. [24-26] for selected examples of TG studies of protons in oxides.

In a different procedure, one replaces all H-containing gases with deuterated equivalents, or vice versa (H/D isotope exchange). The weight change associated with this gives an absolute value of the $\mathrm{H}$-content regardless of defect model. See Refs. [27, 28] for selected examples of TG on isotope exchange.

It should be mentioned that thermogravimetry versus $T, p_{\mathrm{H}_{2}}$, or $p_{\mathrm{H}_{2} \mathrm{O}}$ suffers from many potential sources of error. This includes buoyancy effects (especially of $T$ and $p_{\mathrm{H}_{2}}$ ), dissolution of $\mathrm{H}$ in balance materials such as Pt wires, adsorption of $\mathrm{H}_{2} \mathrm{O}$ on balance parts (especially cold counterweights) and slow changes and equilibria because $\mathrm{H}_{2} \mathrm{O}$ and $\mathrm{D}_{2} \mathrm{O}$ adsorb on apparatus and tubing walls and are changed/exchanged slowly.

A technique used occasionally - providing one has the facility at hand - is cold neutron prompt gamma analysis which gives a measure of hydrogen nuclei in the sample [26]. In secondary ion mass spectrometry (SIMS) we may measure the composition both chemical and isotopic distributions - of a material by sputtering the surface by an ion beam and analyzing the secondary ionized species with a mass spectrometer. The method is thus, in principle, ideal for analyzing $\mathrm{H}$ and $\mathrm{D}$ contents of materials after equilibration and cooling/quenching. It suffers from some problems of calibration and backgrounds in the case of the very lowest masses, and it is for this reason more reliable and sensitive for D than for H. SIMS yields depth profiles and has microscopic lateral resolution, and thus allows analysis of diffusion experiment profiles. See Refs. [29, 30] for examples of SIMS studies on protons in oxides. 
A number of spectroscopic methods are, in principle, well suited and have been applied for analysis of protons in oxides, notably infrared (IR) and Raman [31, 32], and nuclear magnetic resonance (NMR) [33]. However, their application to high temperature proton conductors - where protons are defects rather than structural constituents - is not widespread. The optical techniques suffer from problems of contributions from surfaces and internal interfaces, while small concentrations are troublesome for NMR. Moreover, the strength of these methods is to yield information about the state and position of the hydrogen species, and it would then be particularly important to apply them at high temperatures (one can hardly expect to freeze a proton in place by quenching), which is not easy. It is probable that we will see a development and standardization of these techniques for studies of high temperature proton conductors at high temperatures in the future.

Muons are elementary particles that have the same charge as a proton and $1 / 9$ th of its mass. When introduced in a chemical setting like an oxide, they thus behave like a light hydrogen ion isotope. They can take up an electron and become muonium, a hydrogen-like atomic species. They have short lifetimes, but one may study their oxidation state and environment by muon spin resonance $(\mu \mathrm{SR})$ spectroscopy (an analog to NMR spectroscopy). This has been used to study hydrogen in semiconductors [34] and in some cases in relevant oxides [35].

\subsubsection{Diffusion}

The diffusivity of protons can be studied using so-called pulsed field gradient (PFG) NMR [36], which gives a measure of the local random diffusion. One may alternatively record the diffusion during a transient after changing the chemical activity or the H/D isotope ratio around the sample. For this, one may use gravimetry, electrical properties, or optical spectroscopy. Commonly used today is the analysis by SIMS of the profile after isotope exchange (see above). One may note that the various types of experiments give self-diffusion coefficients, isotope interdiffusion (tracer) coefficients, or chemical diffusion coefficients. The latter often reflects chemical diffusion of water, dominated by the slowest defect - often oxygen vacancies.

\subsubsection{Conductivity}

The conductivity of a material is commonly measured at high temperatures in isotherms or isobars. Alternating current (AC) two-electrode measurements on disks can yield the sample conductivity if the frequency is high enough that the electrode polarisation is eliminated. Impedance spectroscopy can give more information, both to ensure that one is not victim of parasitics or artifacts, and - in some cases - to deconvolute the impedance of the sample into contributions from grain interior, grain boundaries, and electrodes. Impedance spectroscopy can reveal the resistance across grain boundaries, but it cannot give information about parallel conductivity along grain boundaries. 
Figure 1.5 shows a simplified schematic of an equivalent circuit (see, for example, Ref. [37]) representing a mixed-conducting ceramic sample with electrodes, as measured in impedance spectroscopy or transport number studies (below) of a material. One must realize that many possible elements are left out, and the connectivity in the schematic is necessarily over-simplified, and may not serve all situations. This relates, for instance, to electronic conduction across or in grain boundaries. All elements cannot be deconvoluted from one impedance spectrum; one needs additional information or simplifications/assumptions. The trivial aspects of this are, as said above, that parallel grain boundary conduction and grain interior (bulk) conduction cannot be separated - as evident from Fig. 1.5. The less trivial is that the parallel electronic conduction of a mixed conductor assumed to be connected effectively between the electrodes may, in general, not be deconvoluted unambiguously from the rest of the circuit. One may further note that the way Fig. 1.5 is connected, it suggests that bulk and grain boundary resistivities are ionic (e.g., protonic) and requires an electrode (redox) process represented by the so-called Randles circuit to the right, whereas the electronic conduction takes place without series grain boundary or electrode contact resistances. Finally, the figure may represent the total impedance of a mixed-conducting membrane in operation or in a permeation measurement, where the current loops internally through both the electronic and ionic paths, and where the total resistance is that of the sum of the two paths. The Randles part of the figure then must be taken to represent effectively the overall surface redox reactions.

When materials have very high conductivities, one needs to use four-electrode measurements to avoid limitations from the in-plane resistance of the electrode

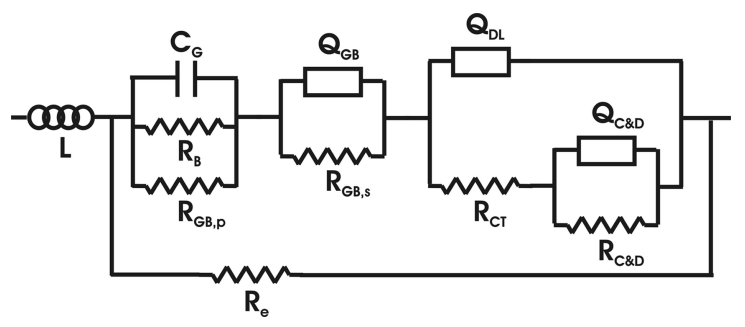

Figure 1.5 Schematic example of a simplified equivalent circuit for a mixed conducting ceramic membrane or sample under investigation of its electrical properties. $L$ is a parasitic inductive element arising from various sources (sample, wires, instrumentation). The leftmost lumped subcircuit $\left(R_{\mathrm{GB}, \mathrm{p}} R_{\mathrm{B}} C_{\mathrm{G}}\right)$ contains the geometrical capacitance, the bulk ionic conductance, and the conduction of ions parallel in the grain boundaries. The next $\left(R_{\mathrm{CB}, \mathrm{s}} Q_{\mathrm{CB}}\right)$ contains the resistance and capacitance across grain boundaries, and where $Q$ is a constant phase element [37], often with $n \approx 3 / 4$ reflecting a dispersion of time constants for the variety of grain boundaries. The rightmost subcircuit

$\left(Q_{\mathrm{DL}}\left(R_{\mathrm{CT}}\left(R_{\mathrm{C} \& \mathrm{D}} Q_{\mathrm{C} \& \mathrm{D}}\right)\right)\right.$ represents the electrodes (or effectively surfaces), and contains double layer capacitance, charge transfer resistance, and chemical and diffusional capacity and transport. The $Q_{D L}$ and $Q_{C \& D}$ constant phase elements often have $n \approx 3 / 4$ and $n \approx 1 / 2$. The $C_{\mathrm{G}}, Q_{\mathrm{GB}}, Q_{\mathrm{DL}}$, and $Q_{\mathrm{C} \& \mathrm{D}}$ typically come out corresponding to capacitances of the order of $10^{-12}, 10^{-9}, 10^{-6}$, and $10^{-3} \mathrm{~F}$ for $\mathrm{cm}$-sized samples. The element $R_{\mathrm{e}}$ represents electronic conductance, here assumed to connect effectively directly between electrodes (surfaces). 
material. This usually becomes important when the measured sample resistance decreases to below around $10 \mathrm{ohm}$. Four-electrode geometries can be applied to bar samples and to disks (van der Pauw method). For mixed conductors, the current electrodes may partly obstruct ionic transport, and the resulting conductivity reflects less ionic contribution than it should. To overcome this, one may apply higher DC voltages or use AC measurements. The van der Pauw method requires, in principle, DC measurements, but AC measurements can be applied if one ensures that a frequency is used where the response does not contain imaginary components.

The total conductivity is:

$$
\sigma=\sum_{\mathrm{i}} \sigma_{\mathrm{i}}=\sum_{\mathrm{i}} z_{\mathrm{i}} F c_{\mathrm{i}} u_{\mathrm{i}}
$$

where $z_{\mathrm{i}}$ is the number of charges of the carrier $i, c_{\mathrm{i}}$ is its concentration (in $\mathrm{mol} \mathrm{m} \mathrm{m}^{-3}$ ) and $u_{\mathrm{i}}$ is its charge mobility in $\mathrm{m}^{2} \mathrm{~V}^{-1} \mathrm{~s}^{-1}$. As mentioned before, the concentration, $c_{\mathrm{i}}$, can be calculated from the mole fraction of the carrier and the molar density of the material: $c_{\mathrm{i}}=[i] d_{\mathrm{M}, \mathrm{i}}$. For diffusing species (ions or ionic defects, including protons, and small polaron electronic carriers) the charge mobility, $u_{\mathrm{i}}$, is given as:

$$
u_{\mathrm{i}}=u_{0, \mathrm{i}} T^{-1} \exp \left(-Q_{\mathrm{m}} / R T\right)
$$

Conductivity measured versus $T$ and $p_{\mathrm{H}_{2} \mathrm{O}}$ can give information about the mobility as well as hydration thermodynamics if one can properly apply the defect model at play and assign the total conductivity to contributions from the relevant charge carriers. Conductivity versus $p_{\mathrm{O}_{2}}$ is less informative in this respect, but can help deconvolute the total conductivity into electronic and ionic parts, as the ionic part (including the protonic) is usually $p_{\mathrm{O}_{2}}$-independent while the electronic ones have $p_{\mathrm{O}_{2}}$ dependences. Refs. [38-40] exemplify typical studies of conductivity of mixed proton conductors.

Conductivity measurements are sometimes done on porous samples to speed up equilibration with the atmosphere. In this case, the measured conductivity should be corrected for porosity, but unfortunately there is no way of doing this accurately for real-world pore distributions and shapes. A commonly used empirical correction for modest porosities is:

$$
\sigma=\frac{\sigma_{\text {measured }}}{d^{2}}
$$

where $d$ is the relative density of the sample.

\subsubsection{Transport Numbers}

The transport numbers, $t_{\mathrm{i}}=\sigma_{\mathrm{i}} / \sigma_{\text {total }}$, are essential in order to calculate individual partial conductivities of different species from the total conductivity measure- 
ment. Traditionally, the transport number of ions in oxides is measured via the open circuit voltage of oxygen concentration cells ("EMF method") or by measuring conductivity with electron- or ion-blocking electrodes.

For proton-conducting materials, the EMF method can be used provided one controls the hydrogen and oxygen activity gradients independently. An EMF measurement with gradient in oxygen only (no gradient in hydrogen) yields the oxygen ion transport number, while a measurement with a gradient only in hydrogen activity yields the proton transport number [41, 42].

In principle, EMF measurements reveal the sign of the hydrogen ion charge carrier. Indications in the literature of negative charge on hydrogen ions under reducing atmospheres - appearing to reflect hydride ion $\left(\mathrm{H}^{-}\right)$transport - are now attributed to diffusion of neutral hydrogen in the materials, polarizing the electrodes and giving rise to false gradients [2].

A proper interpretation of EMF measurements of transport numbers should take into account the polarization of electrodes in the case of ionic transport [43]. This can be obtained from impedance spectra.

Transport numbers can also be obtained from chemical analyses of products during electrochemical pumping. Moreover, the isotope effect on proton conductivity can be used to identify proton conductivity and also, to some extent, to calculate transport numbers quantitatively [44].

One may expect that many hydrogen-permeable oxides investigated will have high electronic conductivities and proton conductivities orders of magnitude lower - much the same as the oxygen ion conductivity is small in oxygen-permeable oxides. In such cases, it is notoriously difficult to measure the transport number of the ionic species by electrical methods, and instead one has to obtain the conductivity of ions indirectly through diffusion measurements, as above, or through permeation measurements as we shall discuss below.

\subsubsection{Other Properties}

Of course, there are more bulk properties of interest than the above parameters related to transport of the fast ions and electrons. Metal cation transport is minor, but still a most crucial parameter, because it eventually leads to membrane walkout, demixing, or decomposition in chemical gradients. Methods used for investigating metal cation diffusion comprise reactivity studies, interdiffusion couples, and tracer studies, using analytical SEM, EPMA, SIMS or radioactivity for the diffusion profile analyses.

Evaporation and chemical stability towards gases and contacting materials are other issues that need investigation. In principle, these can all be calculated from thermodynamic data, but for most materials of interest such data are nonexistent or insufficient.

Mechanical properties are important for real applications. This is a field where, again, we need more data, in particular at high temperatures. In addition to thermal expansion and the problem of thermal cycling, membranes standing in chemical gradients may suffer from chemical expansion, so that one side expands 
relative to the other as a result of a gradient in defect concentrations. This results in stresses and bending of the membrane, and is well known in oxygen separation membranes, where the effects can be large. In proton-conducting oxides, there have been fewer investigations: So far it seems that oxides expand when oxygen vacancies are filled and replaced by protons by hydration, but the effects are rather small [45]. One may investigate this with controlled atmosphere dilatometry or high-temperature X-ray diffraction.

\subsection{2}

\section{Investigation of Surface Kinetics}

The surface kinetics is crucial for a membrane in operation. For oxygen separation membranes, it has proven to be rate limiting in many cases, and has been studied extensively. For proton-conducting materials and membranes, much less has been done, partly because surface kinetics has been less of a problem up to now. Still, we believe membrane materials will be better and thinner, until the surfaces eventually become rate limiting. We, therefore, mention a couple of techniques for studying hydrogen exchange kinetics.

The use of isotopes in studies of surface kinetics is well known from heterogeneous catalysis. By probing the distribution of different isotope-specific gas species as a function of time over different solid catalyst surfaces, the catalytic activity towards specific reactions can be determined. For studies of surface kinetics relevant to hydrogen separation membranes, this would involve the use of gas species containing hydrogen and deuterium and the possible combination of these, for example, $\mathrm{H}_{2}, \mathrm{D}_{2}, \mathrm{HD}, \mathrm{H}_{2} \mathrm{O}, \mathrm{D}_{2} \mathrm{O}$, HDO, etc.

Mass spectrometry (MS) is a convenient experimental technique to determine the time distribution of these species in a gas. Using an experimental assembly designed by Hultquist et al. [46], we have investigated the rate of surface dissociation of $\mathrm{H}_{2}$ and $\mathrm{D}_{2}$ on different proton and mixed electron-proton conductors in the temperature range 200 to $800^{\circ} \mathrm{C}$. A schematic of the experimental assembly is shown in Fig. 1.6. A quadrupole mass-spectrometer (in our case a Balzers, Prisma 200 ) is connected to a small volume of $50 \mathrm{~mL}$ that is heated by a high-temperature furnace (temperatures up to $1100^{\circ} \mathrm{C}$ ). The reaction chamber is connected to volumes where different gases may be pre-mixed before introduction into the reaction chamber. The total pressure in the different chambers is controlled by high precision pressure gauges (Balzers, CMR 60, 0.1-1000 mbar and Baratron, $0.01-100 / 0.1-1000$ mbar). The inlet of gas into the MS is controlled by a leak-valve enabling a very small flow into the MS and, consequently, keeping the total pressure in the reaction chamber virtually constant.

In order to obtain true surface area specific rate constants for the surface kinetics, the real (microscopic) surface area must be taken into account. For powders, the Brunauer, Emmett, Teller (BET) method may be used to determine the true surface area. For dense solid bodies, however, the BET method may be too insensitive, since the overall surface area is relatively low. In such cases, measurements of the surface roughness, for example by atomic force microscopy (AFM), 


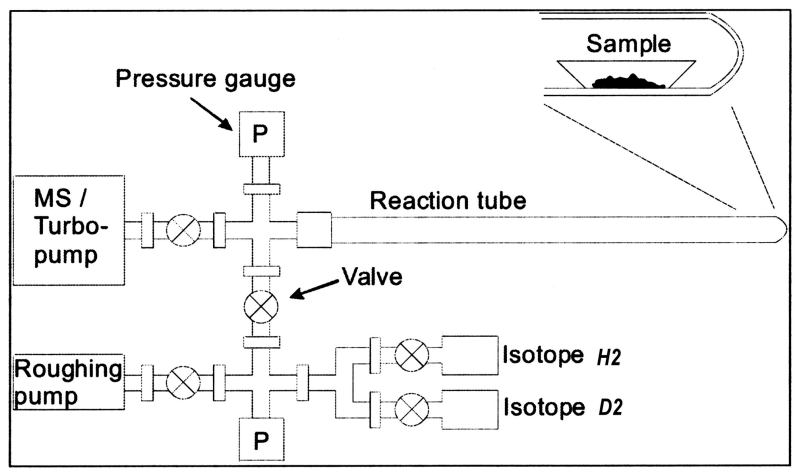

Figure 1.6 Schematic illustration of the experimental set-up for dissociation measurements. From Jens B. Smith, Thesis, Univ. Oslo, 2005.

may form a basis for estimating the effective surface area (as we have done for the data reported below).

Since the oxides do not inherently contain hydrogen, a very simple approach can be used to determine the hydrogen dissociation rate [47, 48]. Starting from mixtures of, for example, $\mathrm{H}_{2}$ and $\mathrm{D}_{2}$, one may follow the evolution of the combined HD molecule and from this calculate the dissociation rate. An example of this type of measurement is shown in Fig. 1.7 where the formation of HD as a function of time starting from a 1:1 mixture of $\mathrm{H}_{2}$ and $\mathrm{D}_{2}$ is compared for undoped and $5 \% \mathrm{Nd}$-doped $\mathrm{CeO}_{2}$. The presence of $\mathrm{Nd}$ increases the rate of dissociation approximately one order of magnitude.

The hydrogen dissociation rate for some oxides of relevance to hydrogen gas separation membranes is presented as a function of the inverse absolute temperature in Fig. 1.8. Data for the $\mathrm{H}_{2}$ dissociation rate on $\mathrm{Ni}$ is included for comparison. As seen from the systems where both doped and undoped materials have been measured, doping generally increases the rate of dissociation.

The data shown in Fig. 1.8 can be used together with information on the partial conductivities to estimate the characteristic length, $L_{C}$, of the oxides if dissociation of hydrogen was the rate-determining step associated with the overall surface kinetics. As we will show in the survey of different mixed conducting materials, $\mathrm{Yb}$-doped $\mathrm{SrCeO}_{3}$ is the only material reported so far where the hydrogen flux has been measured for thicknesses down to only a few $\mu \mathrm{m}$ in thickness. The data from Hamakawa et al. [49] show that the flux remains inversely proportional to the thickness of the membrane down to a thickness of $2 \mu \mathrm{m}$. If we combine the data from Fig. 1.8 with in-house data on the ambipolar proton-electron conductivity of $5 \% \mathrm{Yb}$-doped $\mathrm{SrCeO}_{3}$, we find that the characteristic thickness should be of the order of 0.5 to $1 \mu \mathrm{m}$. One should recognize that the characteristic thickness is not an intrinsic materials property but may well be strongly dependent on the surface microstructure.

Isotope exchange diffusion profiles can also be measured ex situ by SIMS, and can, in principle, reveal surface kinetics in addition to bulk transport. 


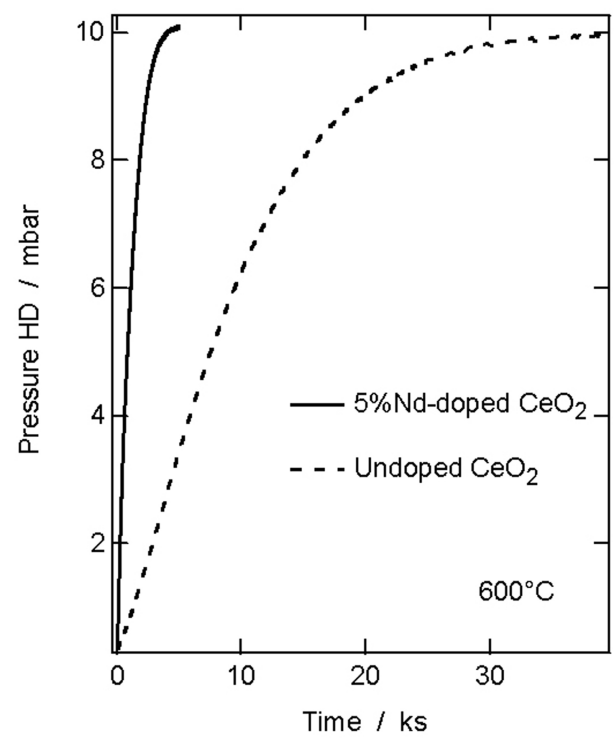

Figure 1.7 The pressure of HD in the reaction chamber as a function of time, starting from a 1:1 mixture of $\mathrm{H}_{2}$ and $\mathrm{D}_{2}$ at 20 mbar total pressure, over undoped and $5 \mathrm{~mol} \% \mathrm{Nd}$-doped $\mathrm{CeO}_{2}$ at $600^{\circ} \mathrm{C}$.

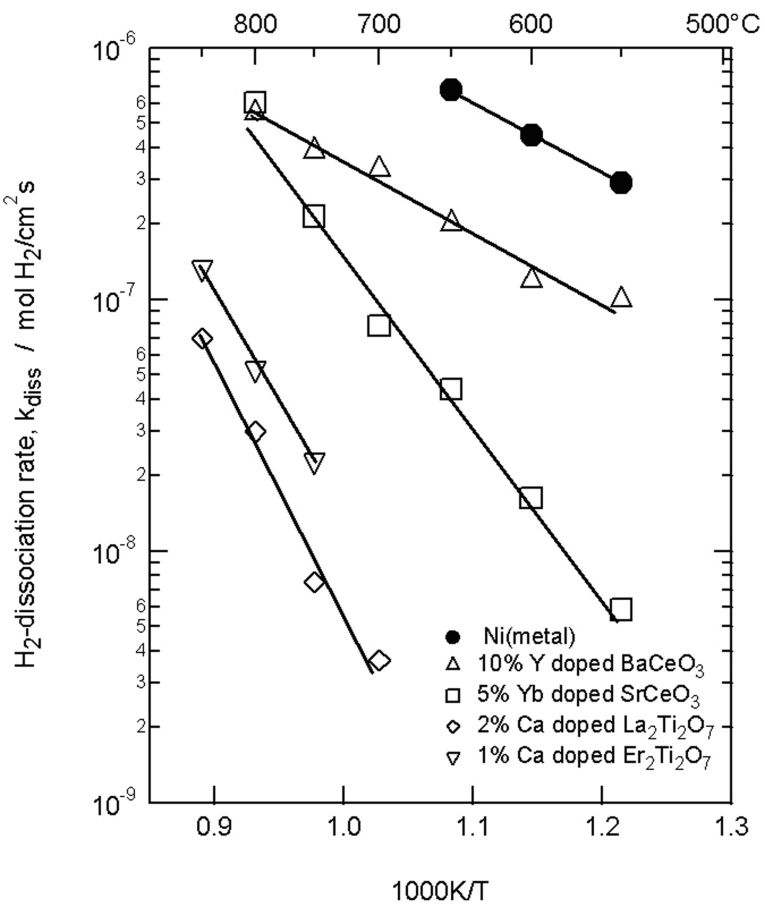

Figure $1.8 \mathrm{H}_{2}$ dissociation rate as a function of the inverse temperature for selected materials, all measured at a total pressure of 20 mbar. 
Transients after step changes in, for example, $p_{\mathrm{H}_{2}}$ or $p_{\mathrm{H}_{2} \mathrm{O}}$ can be followed with conductivity, TG, or spectroscopic methods and can, in principle, reveal chemical diffusion and surface kinetics.

\section{8 .3}

\section{Measurements and Interpretation of Hydrogen Permeation}

It is, of course, necessary to test the hydrogen flux in a real membrane under realistic conditions. This can also give significant information on transport processes in the material. It can even be the only way to measure hydrogen transport in materials where, for example, electronic conductivity is so high that transport number measurements by the EMF method or other electrical methods are impossible.

Setting up measurements for hydrogen permeation in ceramic membranes has some similarities with set-ups for oxygen permeation, but is in most respects more difficult. This arises from notorious leakages of hydrogen through sealing gaskets, support components, and container walls, but also from high backgrounds, high storage capacity and slow exchange by absorption of $\mathrm{H}_{2}$ and adsorption of $\mathrm{H}_{2} \mathrm{O}$, problematic quantitative analyses in some techniques (e.g., mass spectrometry), and ambiguous interpretation of data in some cases.

A permeation experiment is usually performed by setting up a gradient in hydrogen partial pressure and measuring the amount of hydrogen in the permeate off-gas. By assuming a continuously stirred tank reactor (CSTR) model for the membrane sides, the hydrogen partial pressures can be taken to be that of the offgas, and transport parameters such as ambipolar proton-electron conductivity can be calculated from a chosen model and the equations derived for the flux.

Before the flux can be inserted into the equation, it must be corrected for leakages. It is useful to add an inert gas to the feed side hydrogen, so that its content in the permeate reveals the leakage. We may use, for instance, a mixture of $\mathrm{H}_{2}$, $\mathrm{He}$, and $\mathrm{N}_{2}$ as feed gas, and $\mathrm{Ar}$ as permeate sweep gas. If the ratio of $\mathrm{He}$ and $\mathrm{N}_{2}$ in the permeate is the same as in the feed, then the leakage is macroscopic, and the $\mathrm{H}_{2}$ leaked can be calculated from the ratio of $\mathrm{H}_{2}$ and $\mathrm{He}$ in the feed gas and the He leakage rate. If the He to $\mathrm{N}_{2}$ ratio is changed, then one may assume Knudsentype or other type of leakage, and the hydrogen leakage cannot be calculated so easily. In any case, this method deals with open leakages, and may not pick up hydrogen diffusion through seal gasket materials, etc.

Deuterium on the feed side may be used to better separate permeated hydrogen from background sources.

Water vapor may play an important role. Firstly, water vapor is in many cases the actual source of the protons in the membrane material. Thus, a hydrogenpermeable oxide may simply not work in dry gases. Secondly, water vapor determines the oxygen activity, and the membrane may be unstable in dry hydrogen. Thirdly, the water vapor may be used to set up or to balance out an oxygen activity gradient. It may also affect the electronic conductivity. Normally, it is good practice 
to operate with wet gases on both sides of the membrane so that conditions are well-defined.

Once a leakage-corrected flux is obtained, one should ascertain that it arises from hydrogen permeation and not other transport processes. For instance, a possible mistake is to analyze and find hydrogen in the permeate and ascribe it to hydrogen permeation, while the real source is oxygen transport in the opposite direction: This splits water vapor on the permeate side and leaves hydrogen. Only careful analysis of water levels can differentiate the two cases. One may try to circumvent the problem by using a dry permeate side sweep gas, but even impurities or leakages of water may be more than enough to form hydrogen by oxygen transport.

For experimental studies we refer to the references in the literature section below.

\section{9}

\section{Hydrogen Permeation in Selected Systems}

\subsection{1}

\section{A Few Words on Flux and Permeability}

Before we move on to review results of hydrogen permeation in various systems, we dwell on some terms of flux and permeability.

The process of permeation through a membrane gives rise to a flux, or permeation. Commonly used units are $\mathrm{mol} \mathrm{s}^{-1}$ and $\mathrm{mL}_{\mathrm{n}} \mathrm{min}^{-1}=\mathrm{mL} \mathrm{min}^{-1}(\mathrm{STP})$. In order to obtain (area specific) flux densities we divide by area and get, for example, units of $\mathrm{mol} \mathrm{s}^{-1} \mathrm{~cm}^{-2}$ or $\mathrm{mL}_{\mathrm{n}} \mathrm{min}^{-1} \mathrm{~cm}^{-2}$.

In traditional membrane science, the permeability is the specific materials property coefficient that relates the pressure or concentration difference over the membrane to the flux density. The permeance is similarly the area-specific coefficient obtained after division by the membrane thickness. Typical units would be mol s $\mathrm{cm}^{-1} \mathrm{~Pa}^{-1}$ for permeability and $\mathrm{mol} \mathrm{s}^{-1} \mathrm{~cm}^{-2} \mathrm{~Pa}^{-1}$ for permeance.

For dense membranes it is more complicated, since the materials property itself can be pressure dependent, and the flux gets various pressure dependences depending on defect structure. It is therefore common to use the terms permeance and permeability for the flux including the actual pressures involved. Permeance is then the same as flux, typically given in area specific values like flux density, with units like mol s $\mathrm{cm}^{-2}$. Permeability is used for the materials specific - thickness independent - flux density obtained by multiplying by the membrane thickness, and with units of typically $\mathrm{mol} \mathrm{s}^{-1} \mathrm{~cm}^{-1}$ or $\mathrm{mL}_{\mathrm{n}} \mathrm{min}^{-1} \mathrm{~cm}^{-1}$.

Alternatively, the permeance and permeability of a dense membrane can be expressed as a coefficient of flux density per unit pressure to a power depending on defect model (for instance, from the examples we have seen in Section 1.4, 1/2, $1 / 4,0,-1 / 2$, and $-1 / 4)$. We will see examples of several ways of using these units 
later - the intention here is to prepare the reader to be careful when reading and quoting fluxes and permeabilities.

\subsection{2}

\section{Classes of Membranes}

Inorganic gas-separation membranes may, in gross terms, be divided into two major categories, dense membranes as we have dealt with above, and porous membranes. The selectivity of a porous membrane is simply based on the pore size in the barrier layer. Membranes may, as such, based on the size of the gaseous molecules, be designed to separate specific gases or mixtures of gases. Depending on the porosity and the dimension of the pores, different transport mechanisms may apply: Knudsen diffusion, surface diffusion, molecular sieving, viscous flow, activated diffusion and capillary condensation. The driving force for separation of the desired gas species is normally a large (often orders of magnitude) difference in total pressure between the feed and the permeate side.

State-of-the-art micro-porous membranes are based on silica, with sufficiently small pores, 2-10 $\AA$, to be selective towards hydrogen separation. One of the major problems with silica membranes under hydrothermal conditions is physical stability. Evaporation of silica-containing species is detrimental to long-term permselectivity and restricts the operation of these membranes to temperatures below $600^{\circ} \mathrm{C}$. Hydrogen permeances of $>1 \times 10^{-6} \mathrm{~mol} \mathrm{~m}^{-2} \mathrm{~s}^{-1} \mathrm{~Pa}^{-1}$ with $\mathrm{H}_{2} / \mathrm{CO}_{2}$ permselectivity in the range 80-100 have, for instance, been measured with single deadend tubular micro-porous silica membranes for temperatures higher than $300^{\circ} \mathrm{C}$ and with 4 bar pressure difference. These membranes were reported to be thermally stable for at least $2000 \mathrm{~h}$ at temperatures between 200 and $400{ }^{\circ} \mathrm{C}$ [50].

Dense inorganic membranes for hydrogen separation may consist either of a metal or an alloy, a ceramic (oxide), or a mixture of both, a so-called cermet. One may also envisage a mixture of two ceramics ("cercer"). In the following, we discuss literature on hydrogen permeability in some of these classes of dense membranes.

\section{9 .3}

\section{Mixed Proton-Electron Conducting Oxides}

We have seen that the hydrogen flux across a ceramic mixed proton-electron conductor is proportional to the ambipolar protonic-electronic conductivity (Eq. (1.36)), that is, it is determined mainly by the protonic or electronic conductivity, whichever is smaller. Therefore, a prerequisite for a good candidate membrane material is a combination of both high electronic and high protonic conductivity. To date, not many materials are known to meet both these criteria. In addition, other material aspects must also be taken into account in evaluation of possible membrane candidates: The different cations of the oxide must be stable under sufficiently reducing conditions (high $\mathrm{H}_{2}$ pressures and high temperature). Because the materials must withstand relatively large chemical potential gradi- 
ents, one should consider whether chemical de-mixing of ternary oxides may occur. The materials, furthermore, must show good mechanical stability with low creep rates, since they, in addition to activity gradients, also may experience total pressure gradients and gravity. Furthermore, depending on the reaction environment, the materials must be stable toward degradation caused by gas species other than hydrogen. This may typically be $\mathrm{CO}_{2}$ and other carbon-containing species as major constituents of the feed gas, and $\mathrm{H}_{2} \mathrm{~S}$ or other S-containing species as impurities.

These are all among the important aspects when designing a hydrogen membrane reactor to serve at intermediate and high temperatures. However, because much of the focus so far in the literature has been on developing materials with a sufficiently high permeation of hydrogen to become commercially viable - that is, exhibiting high ambipolar hydrogen-electron conductivity - the focus of this survey is primarily on transport properties.

State-of-the-art proton conductors comprise acceptor-substituted perovskites, such as the barium-based ones $\left(\mathrm{BaCeO}_{3}, \mathrm{BaZrO}_{3}\right.$, etc.) which exhibit proton conductivities in excess of $0.01 \mathrm{~S} \mathrm{~cm}^{-1}$ [51-55] and strontium-based ones $\left(\mathrm{SrCeO}_{3}\right)$ with somewhat lower conductivities. Both $\mathrm{BaCeO}_{3}$ and $\mathrm{BaZrO}_{3}$ are almost pure ionic conductors, and the electronic conductivity would, as such, rate limit the $\mathrm{H}_{2}$ flux across membranes of these materials [56].

$\mathrm{BaPrO}_{3}$ and $\mathrm{BaTbO}_{3}$ also possess perovskite-related structures and exhibit considerable proton conductivity [57-60]. Because Pr and Tb show mixed valence (III/ IV), one may expect high electronic conduction, and the materials indeed exhibit high p-type electronic conductivity under oxidizing conditions. However, the chemical stability of these compounds is poor even under mildly reducing conditions ( $\mathrm{Ar}$ or $\mathrm{N}_{2}, 1$ to $10 \mathrm{ppm} \mathrm{O}_{2}$ ). Balachandran et al. [61] improved the stability of $\mathrm{BaPrO}_{3}$-based compounds by substituting $\mathrm{Zr}$ and $\mathrm{Y}$ for $\mathrm{Pr}$, forming $\mathrm{BaZr}_{x} \mathrm{Pr}_{0.8-x} \mathrm{Y}_{0.2} \mathrm{O}_{3-\delta}$. It was observed that the total conductivity was considerably higher than for BCY (barium cerium yttrium oxide). However, because only total conductivities were determined, it is difficult to evaluate the prospects of these materials as membrane candidates.

Different types of doping have been tried in order to increase the partial electronic conductivity in $\mathrm{BaCeO}_{3}$. Matsumoto et al. [62-64] recently reported that $\mathrm{BaCeO}_{3}$ and $\mathrm{SrZrO}_{3}$ co-doped with $\mathrm{Ru}$ and $\mathrm{Y}$ exhibit enhanced mixed proton-electron conductivity. The electronic conductivity, based upon X-ray absorption spectroscopy, was concluded to be p-type also under reducing conditions. The flux density of hydrogen was measured at $800^{\circ} \mathrm{C}$, and for a $0.5 \mathrm{~mm}$ thick membrane of $\mathrm{BaCe}_{0.8} \mathrm{Y}_{0.1} \mathrm{Ru}_{0.1} \mathrm{O}_{3-\delta}$ it was found to be $0.07 \mu \mathrm{mol} \mathrm{cm} \mathrm{cm}^{-2} \mathrm{~s}^{-1}\left(0.005 \mathrm{~mL}_{\mathrm{n}} \mathrm{min}^{-1} \mathrm{~cm}^{-2}\right)$ with a ratio between the hydrogen pressures of the two sides of approximately 500 (Fig. 1.9). The values for the $\mathrm{SrZrO}_{3}$ materials were approximately a factor of two lower. Compared with other literature data on similar perovskites, the presence of $\mathrm{Ru}$ appears to have increased the permeability one to two orders of magnitude.

Acceptor-doped $\mathrm{SrCeO}_{3}$ is, without doubt, the mixed electron-proton conducting oxide system most studied, both with respect to actual measurements of 


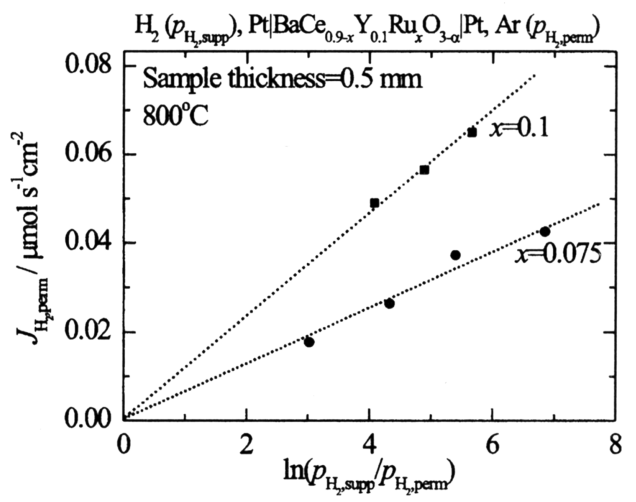

Figure 1.9 Flux density through $0.5 \mathrm{~mm}$ thick $\mathrm{Y}+$ Ru-doped $\mathrm{BaCeO}_{3}$ membranes (two different Ru contents) versus logarithm of feed- to permeate-side hydrogen ratio. From Matsumoto et al. [63].

hydrogen fluxes, and to estimation of hydrogen fluxes based on models and conductivity data, and modeling of defect properties and dopant behavior [20, 65-67].

Acceptor-doped $\mathrm{SrCeO}_{3}$ is among the best proton conductors, with only a factor of $\sim 2$ lower proton conductivity than $\mathrm{BaCeO}_{3}$-based materials. Both these oxides are predominantly protonic conductors below $600-700^{\circ} \mathrm{C}$. The $\mathrm{SrCeO}_{3}$ has the advantage that the n-type electronic transport number is higher, and the oxygen ion transport number is lower relative to those for $\mathrm{BaCeO}_{3}$ at high temperatures. Hydrogen permeation in $\mathrm{SrCeO}_{3}$-based systems is therefore superior to that in $\mathrm{BaCeO}_{3}$-based systems. However, the partial electronic conductivity appears still to remain rate limiting for hydrogen permeability. Different doping strategies have been followed to increase the electronic as well as the ionic conductivity of $\mathrm{SrCeO}_{3}$. Cermets with $\mathrm{SrCeO}_{3}$ have also been tested [68, 69]. Aliovalent cations that have been tested for the purpose of increasing the conductivity include $\mathrm{Y}$ [70, 71], $\mathrm{Yb}[72], \mathrm{Tm}$ [73], and $\mathrm{Eu}[74,75]$.

Hydrogen fluxes through $\mathrm{SrCeO}_{3}$-based systems have recently been measured for asymmetric membranes [49, 76]. Figures 1.10 and 1.11 illustrate from these two investigations the variations in the flux with different upstream pressures of hydrogen for different membrane thicknesses. The maximum flux was reported by Hamakawa [49] for a $2 \mu \mathrm{m}$ film. Operated with $0.2 \mathrm{~atm} \mathrm{H}_{2}$ on the feed side and He on the permeate side, it gave a hydrogen flux density of $\sim 15 \mathrm{~mL}_{\mathrm{n}} \mathrm{min}^{-1} \mathrm{~cm}^{-2}$ at $950 \mathrm{~K}$. The variation in the hydrogen flux with the thickness of the membranes - from the study of Hamakawa - is shown in Fig. 1.12. The flux is proportional to the inverse thickness of the membrane which corresponds to bulk diffusion limitations. On this basis it was concluded that surface kinetics does not affect the rate-limiting step of hydrogen permeation $[49,76]$.

Because the temperature dependence of the hydrogen flux in $\mathrm{SrCeO}_{3}$ exhibits an essentially straight-line Arrhenius behavior, the electronic conductivity is 


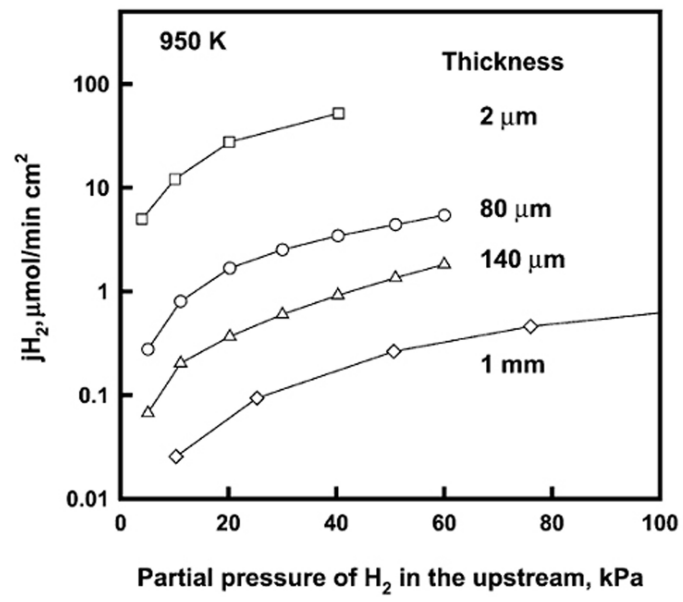

Figure 1.10 Hydrogen flux across membranes of $5 \%$ Yb-doped $\mathrm{SrCeO}_{3}$ as a function of the partial pressure of hydrogen in the feed gas. From Hamakawa et al. [49].

believed to be limiting for the permeability at least up to temperatures of $1050^{\circ} \mathrm{C}$ [77]. This role of the electronic conductivity is also indicated by the hydrogen permeation's dependence on the oxygen activity in the permeate stream as shown here in Fig. 1.13 from the investigation of Cheng et al. [76].

Although acceptor-doped $\mathrm{SrCeO}_{3}$ shows the highest reported hydrogen fluxes so far, long-term use of this material in industrial applications may be difficult

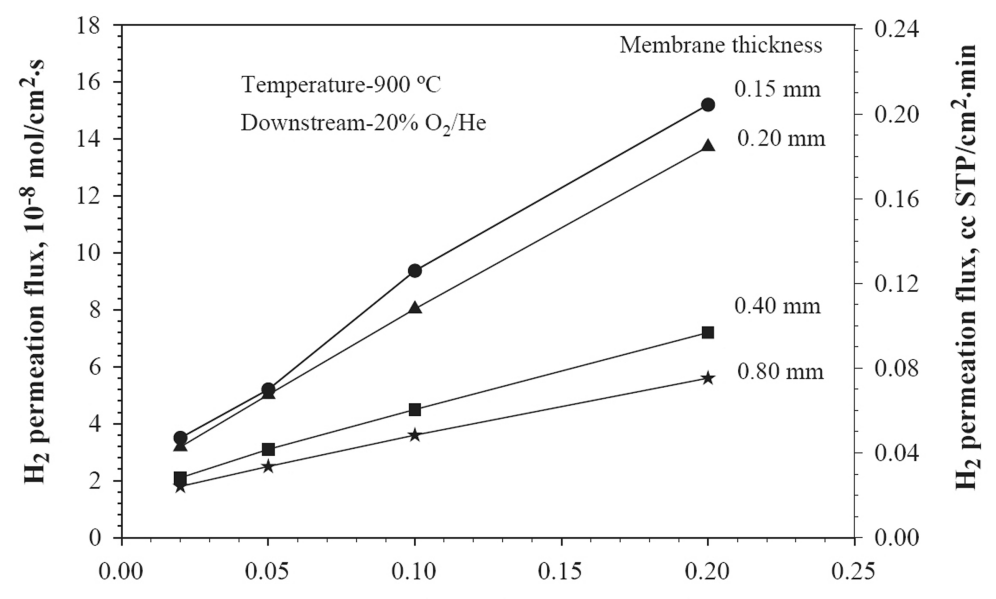

Upstream $\mathrm{H}_{2}$ partial pressure, atm

Figure 1.11 Hydrogen flux across membranes of $5 \%$ $\mathrm{Tm}$-doped $\mathrm{SrCeO}_{3}$ as a function of the amount of hydrogen in the feed gas. From Cheng et al. [76]. 


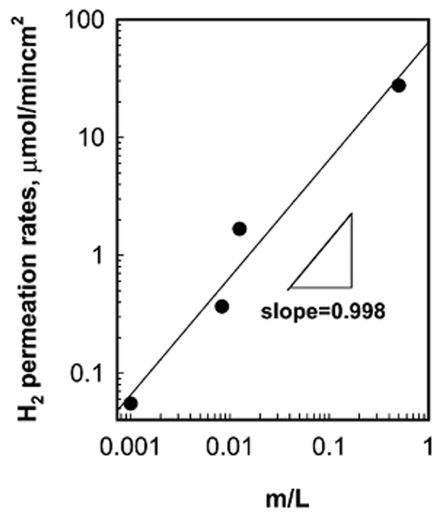

Figure 1.12 Hydrogen flux across a $5 \%$ Yb-doped $\mathrm{SrCeO}_{3}$ as a function of the inverse thickness (in micrometers) of the membrane (950 K). From Hamakawa et al. [49].

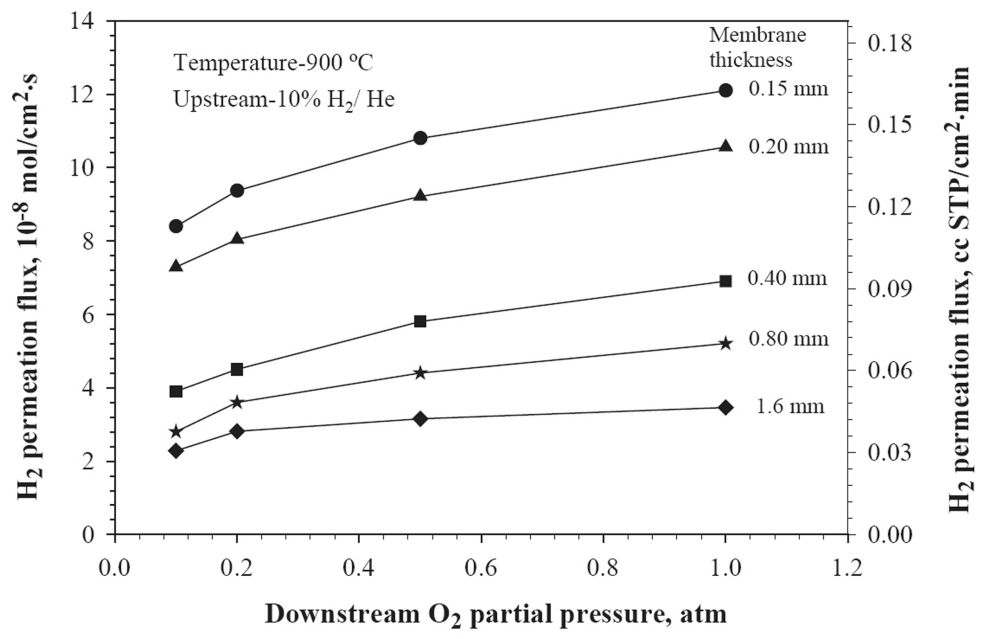

Figure 1.13 Hydrogen flux as a function of the amount of oxygen in the sweep gas for $5 \% \mathrm{Tm}$-doped $\mathrm{SrCeO}_{3}$. From Cheng et al. [76].

because of its reactivity towards even low concentrations of $\mathrm{CO}_{2}$ [78]. One should also note that $\mathrm{SrCeO}_{3}$ becomes unstable under very reducing conditions, for example dry $\mathrm{H}_{2}$ at $1000^{\circ} \mathrm{C}$ [79]. Therefore, the search for new materials combining high proton and electron transport continues with emphasis on stability issues, in particular towards $\mathrm{CO}_{2}$ and other carbon-containing species. We have recently investigated a number of oxide systems expected to combine these properties. Some of the data from these studies are briefly summarized below.

Acceptor-doped rare-earth sesquioxides were one of the first classes of non-perovskite structured oxides that were observed to exhibit proton conductivity [80, 
81]. The conductivity behavior of these oxides was characterized over large ranges of temperature and oxygen activities. Terbium sesquioxide, $\mathrm{Tb}_{2} \mathrm{O}_{3}$, is the only compound examined so far that exhibits both electronic (p-type) and protonic conductivity in hydrogen atmospheres at high temperatures [82]. Hydrogen permeation would be governed by the proton conductivity, which at $900^{\circ} \mathrm{C}$ is approximately $5 \times 10^{-5} \mathrm{~S} \mathrm{~cm}^{-1}$ (5\% of the total conductivity) and would yield a permeability of $3 \times 10^{-5} \mathrm{~mL}_{\mathrm{n}} \mathrm{min}^{-1} \mathrm{~cm}^{-1}$.

With the requirement of electronic conductivity, oxides containing cations with mixed valence and, in particular, reducible cations are preferable. Oxides containing transition metals are therefore appropriate alternatives. There are indications based on conductivity measurements that $\mathrm{TiO}_{2}$ could be a possible candidate [83], but no direct measurements of hydrogen permeability have been reported. Titanates, in general, however, are interesting because there are a number of materials classes that accommodate oxygen vacancies and may dissolve protons.

Among these, the pyrochlore-structured rare-earth titanates $\left(\mathrm{Ln}_{2} \mathrm{Ti}_{2} \mathrm{O}_{7}\right)$ doped with Ca have been studied recently [40,84]. The electronic conductivity predominated under reducing conditions, but with a significant contribution from protons. The highest flux was observed for $1 \%$ Ca-doped $\operatorname{Er}_{2} \mathrm{Ti}_{2} \mathrm{O}_{7}$ [85]; at $900{ }^{\circ} \mathrm{C}$ with $0.5 \mathrm{~atm} \mathrm{H}_{2}$ in the feed and $\mathrm{He}$ as sweep gas, the permeability was $2 \times 10^{-4} \mathrm{~mL}_{\mathrm{n}} \mathrm{min}^{-1} \mathrm{~cm}^{-1}$. This is a factor of two lower than for $5 \% \mathrm{Yb}$-doped $\mathrm{SrCeO}_{3}$ measured under corresponding reaction conditions [86]. One should note here that these measurements were performed using wet sweep gas and that the value for $\mathrm{Er}_{2} \mathrm{Ti}_{2} \mathrm{O}_{7}$ may be too high, due to splitting of water at the permeate side. Acceptor-doped $\mathrm{La}_{2} \mathrm{Zr}_{2} \mathrm{O}_{7}$ is another pyrochlore-structured oxide that has been found to exhibit proton conduction $[87,88]$. Although the proton conductivity in this material is slightly higher than for the titanates, the conductivity of the zirconate is essentially ionic, even in wet hydrogen. Thus, the ambipolar hydrogen electron conductivity becomes correspondingly low.

Increasing the valence of the B-site cation by going to the Group 5 elements, oxides of vanadium are probably too difficult to handle because some of these compounds are rather volatile and have a very complex chemistry. Niobates and tantalates, on the other hand, are of more interest. On the basis of empirical relations between material properties and proton dissolution, double oxides with rare earths as the A-site cations are, again, possible alternatives. Here there are several possible stoichiometries with different crystal structures. The compounds $\mathrm{La}_{3} \mathrm{NbO}_{7}$ [89] and $\mathrm{LnNbO}_{4}$ and $\mathrm{LnTaO}_{4}$ ( $\mathrm{Ln}=$ rare earth) [90], have been found to exhibit considerable proton conductivity. The maximum value, $1 \times 10^{-3} \mathrm{~S} \mathrm{~cm}^{-1}$, was reported for $1 \% \mathrm{Ca}$-doped $\mathrm{LaNbO}_{4}$ and represents the highest proton conductivity measured so far for materials stable under $\mathrm{CO}_{2}$. However, because these classes of materials are almost pure ionic conductors, the ambipolar conductivity would be more than two orders of magnitude lower. Permeabilities would be correspondingly low, $<1 \times 10^{-5} \mathrm{~mL}_{\mathrm{n}} \mathrm{min}^{-1} \mathrm{~cm}^{-1}$. Possibilities to increase the electronic conductivity here would be to substitute a more easily reducible cation on the B-site, for example, Ti. 
$\mathrm{La}_{6} \mathrm{WO}_{12}$ was shown by Shimura et al. [91] to exhibit high proton conductivity. We have recently characterized the electrical conductivity across the $\operatorname{Ln}_{6} \mathrm{WO}_{12}$ series and found a corresponding behavior. In addition, these oxides also exhibit considerable n-type electronic conductivity [92]. Also, $1 \%$ Ca-doped $\mathrm{La}_{6} \mathrm{WO}_{12}$ has in preliminary tests shown relatively high $\mathrm{H}_{2}$ permeabilities, $\sim 0.001 \mathrm{~mL}_{\mathrm{n}} \mathrm{min}^{-1} \mathrm{~cm}^{-1}$, that is, values approximately one order of magnitude higher than for the pyrochlore titanates and more than a factor of two higher than for $5 \% \mathrm{Yb}$-doped $\mathrm{SrCeO}_{3}$. Because $\mathrm{WO}_{3}$ is known to be volatile and because W forms carbides rather easily, the stability of this class of oxides in membrane applications must be considered critically.

\section{9 .4}

\section{Cermets}

Cermets for hydrogen separation consist of a ceramic and a metallic phase contiguous in a dense matrix. In a cermet, one may combine one state-of-the-art pure proton conductor with a highly electron-conducting metallic phase, and thereby circumvent the problem of having both electronic and ionic conduction in one and the same oxide.

Because both metals and oxides alone may transport hydrogen, different combinations with respect to functional properties of both phases have been tried, for example, (i) a metal with low hydrogen conductivity in combination with a highly proton-conductive oxide, (ii) a metal or an alloy with high hydrogen permeability (e.g., $\mathrm{Pt}, \mathrm{Pd}, \mathrm{Pd} / \mathrm{Ag}, \mathrm{Pd} / \mathrm{Cu}, \mathrm{Ni}, \mathrm{Nb}, \mathrm{Ta}, \mathrm{Nb}, \mathrm{V}$ ) together with a ceramic of low hydrogen permeability, and, finally, (iii) a combination where both the metallic and the ceramic phases conduct hydrogen (as protons for the ceramic and hydrogen for the metallic part). Balachandran et al. [93] have investigated the properties of these three combinations with respect to selectivity, permeability and mechanical stability. When a pure proton-conducting oxide constitutes the oxide phase, it is required that the content of metal be high enough to ensure sufficient electronic transport in the metallic phase to maintain the hydrogen flux at its maximum (as defined by the maximum in proton conductivity). Generally, this is satisfied if the metallic phase is percolating through the membrane. This type of membrane (i) has been tested with BCY as the ceramic matrix. It was observed that the hydrogen flux across the composite increases significantly as compared to a fully ceramic BCY membrane. For the combination in which both matrices exhibit $\mathrm{H}_{2}$ permeability (iii), the flux increases even more. It was observed that most of the hydrogen was transported through the metallic phase [94]. Unfortunately, these types of membranes exhibited poor mechanical stability.

From the above evaluation, it seems that a good alternative may be a matrix of a metallic phase with high hydrogen permeability strengthened by a dispersion of ceramic particles (alternative ii). For this class of materials, flux densities have been measured to be of the order of $10-30 \mathrm{~mL}_{\mathrm{n}} \mathrm{min}^{-1} \mathrm{~cm}^{-2}$ using $1 \mathrm{~atm}$ total pressure with $\mathrm{H}_{2}$ as feed gas and $\mathrm{He}$ as sweep gas. In general, the flux in these materials increases with $p_{\mathrm{H}_{2}}^{1 / 2}$ in the feed gas and is inversely proportional to the thick- 


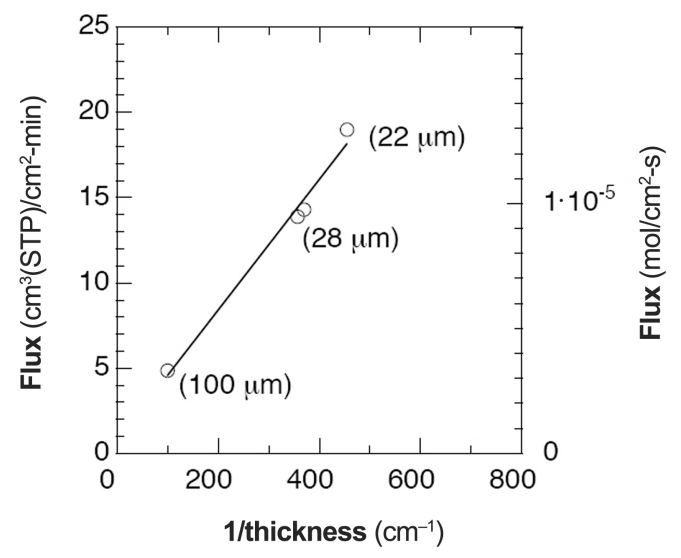

Figure 1.14 Hydrogen flux through a cermet measured at $900^{\circ} \mathrm{C}$ as a function of the inverse thickness of the membrane $\left(1 \mathrm{~atm} \mathrm{H}_{2}\right.$ as feed gas and $\mathrm{He}$ as sweep gas). From Balachandran et al. [93].

ness of the membrane down to at least $22 \mu \mathrm{m}$ (cf. Fig. 1.14) [93]. In this range, the flux is obviously governed by solid-state diffusion and surface kinetics is not rate limiting. The flux has also been shown to increase with temperature up to $900^{\circ} \mathrm{C}$. If the data discussed above are extrapolated to high-pressure feed streams ( $20 \mathrm{~atm})$, flux densities of the order of $200 \mathrm{~mL}_{\mathrm{n}} \mathrm{min}^{-1} \mathrm{~cm}^{-2}$ may be reached. Stability towards feed gas impurities was also studied, for example, for $\mathrm{H}_{2} \mathrm{~S}$ levels of $400 \mathrm{ppm}$, and the $\mathrm{H}_{2}$ flux was stable for at least $700 \mathrm{~h}$ under such conditions.

\section{9 .5}

\section{Permeation in Other Oxide Classes and the Possibility of Neutral Hydrogen Species}

Nigara et al. have reported hydrogen permeation in fluorite-structured materials, such as pure and acceptor-doped $\mathrm{CeO}_{2}$ ceramics [95, 96] and Y-stabilised $\mathrm{ZrO}_{2}$ (YSZ) single crystals [97]. The hydrogen permeation has been measured up to very high temperatures $(1800 \mathrm{~K})$ under reducing conditions. Permeabilities were fairly low; at $1400^{\circ} \mathrm{C}$ and with wet diluted $\mathrm{H}_{2}$ versus $\mathrm{Ar}$ they were of the order of $10^{-6} \mathrm{~mol} \mathrm{H} \mathrm{h}^{-1} \mathrm{~cm}^{-1}$ for undoped and acceptor-doped $\mathrm{CeO}_{2}$ and $10^{-7} \mathrm{~mol} \mathrm{H} \mathrm{h}^{-1} \mathrm{~cm}^{-1}$ for YSZ single crystals. The activation energies are in general above $1 \mathrm{eV}$. The results were suggested to reflect ambipolar transport of protons and electrons. These materials are oxides that are otherwise mostly known for their oxygen ion conductivity and use as electrolytes in solid oxide fuel cells. The appearance of the hydrogen permeation in these materials coincides with conditions for which a negative transport number of hydrogen has been measured in concentration cells, attributed tentatively to polarization of electrodes or surfaces by transport of neutral hydrogen species through the materials rather than protons, as outlined earlier.

Serra et al. [98] recently reported hydrogen permeation through commercial $\mathrm{Al}_{2} \mathrm{O}_{3}$ tubes and interpreted the results in terms of a flux of neutral hydrogen 
atoms. A typical flux density for a $2.75 \mathrm{~mm}$ thick wall and $1 \mathrm{~atm} \mathrm{H}_{2}$ versus vacuum at $1400^{\circ} \mathrm{C}$ was $4 \times 10^{-8} \mathrm{~mol} \mathrm{H}_{2} \mathrm{~s}^{-1} \mathrm{~m}^{-2}$. Others have reported mixed proton transport in the same material [99], providing an illustration of the uncertainty of the relative roles of various oxidation states of hydrogen. If we move on to even more covalent oxides (still with large band gaps) such as $\mathrm{SiO}_{2}$, it appears that neutral species take over even more in permeation; both $\mathrm{H}$ and $\mathrm{H}_{2} \mathrm{O}$ are believed to diffuse in $\mathrm{SiO}_{2}$ much as neutral entities $[100,101]$.

There has also been interest in $\mathrm{Ca}_{12} \mathrm{Al}_{14} \mathrm{O}_{33}$ recently, in which hydrogen is reported to dissolve in the sub-nanometer cages in the structure in $\mathrm{H}_{2}$ atmospheres at, for example, $1300^{\circ} \mathrm{C}$ and to disproportionate into hydride ions and protons [3]. The hydrogen gives rise to electronic conduction at room temperature after UV irradiation. The cages can host a variety of species, and the material exhibits both oxygen ion conduction [102] and large water uptake [103] at high temperatures, in addition to the said hydrogen uptake. Hydrogen permeation may thus take place by various mechanisms and is one of the many properties of this fascinating material presently under investigation.

\section{9 .6}

\section{Comparison with Metals}

Several metals and alloys have been shown to exhibit high permeabilities of hydrogen. The noble metals, in particular, Pd and alloys of Pd-PdAg and -PdCu have attracted much attention. Compared to pure Pd, some Pd alloys show a higher permeance in addition to better resistance to hydrogen embrittlement, better tolerance to gaseous impurities, and lower materials costs.

The most pronounced problem of these materials is, nevertheless, the poisoning by impurities in the feedstream, and this often limits their applicability. Alloys of $\mathrm{Pd}-\mathrm{Ag}$ are particularly prone to poisoning by sulfur. Other gases such as $\mathrm{CO}$ and $\mathrm{H}_{2} \mathrm{O}$ can also be detrimental to long-term membrane performance. The highest measured permeabilities of these materials are of the order of $10^{-9}$ to $10^{-8} \mathrm{~mol} \mathrm{H}_{2} \mathrm{~m}^{-1} \mathrm{~s}^{-1} \mathrm{~Pa}^{-n}$, where typically $n=0.5$ [104-106].

Membranes based on the noble metals have the disadvantage of very high materials costs. Therefore, other less noble metals and their alloys provide interesting alternatives. Here, the Group 4 and 5 elements including Nb, Ta, V, and Zr exhibit the best performance. In comparison to $\mathrm{Pd}$, Ta is approximately one order of magnitude cheaper and is reported to exhibit hydrogen permeabilities approximately one order of magnitude higher [107]. Membranes based on the Group 4 and 5 metals have shown permeabilities of over $400 \mathrm{~mL}_{\mathrm{n}} \mathrm{min}^{-1} \mathrm{~cm}^{-1}$ at $440{ }^{\circ} \mathrm{C}$ with a pressure difference of 33 bar [108]. 


\subsection{0}

Summary

Dense inorganic membranes with mixed protonic-electronic conduction - typically oxides - find prospective use in high-temperature hydrogen separation membranes, especially where process integration is possible. Uses in fossil-fueled power plants with $\mathrm{CO}_{2}$ sequestration have been exemplified. The state-of-the-art materials in this class are too basic and suffer from stability problems, while the more stable materials have ambipolar conductivities on the low side. We thus need to identify new stable materials with high combined electronic and protonic conduction. On the way, a better understanding of the protonation thermodynamics, general defect structures, and transport of protons in the candidate materials is in demand. As bulk properties are improved, similar advancement in understanding and characterization of surface kinetics will become mandatory. Research in this field is thus needed, but involves rather complex defect models and transport equations. We have in this chapter indicated simple defect models and derived some transport equations as an incentive and help in this direction. We need good measurement procedures, especially for permeation studies, and we have indicated some of these, and their pitfalls. Finally, we use the opportunity to emphasize the importance of the materials' chemical and thermal stability, mechanical strength, and the ceramic engineering to make dense, thin membranes on porous supporting substrates - all needed to arrive at long-lived high-performance membranes for hydrogen separation at high temperatures.

\section{Acknowledgements}

The Research Council of Norway (RCN), Norwegian industries Norsk Hydro ASA and Statoil ASA, and the EU/GROWTH programme have funded and supported several projects in the authors' group within the general theme of this contribution. The authors are grateful for the insight gained through these projects and the collaboration with all the project partners. 


\section{References}

1 T. Norby, Y. Larring, Solid State Ionics 2000, 136-137, 139-148.

2 T. Norby, M. Widerøe, R. Gløckner, Y. Larring, Dalton Trans. 2004, 19, 30123018.

3 K. Hayashi, S. Matsuishi, T. Hosono, T. Kamiya, M. Hirano, H. Hosono, Nature 2002, 419, 462-465.

4 K. Åsen, B. Vigeland, T. Norby, Y. Larring, T. Mejdell, in Proceedings of the 7 th International Conference on Greenhouse Gas Control Technologies (GHGT-7), Vancouver, Canada, Sept. 5-9, 2004, Vol. 1: Peer-Reviewed Papers and Plenary Presentations, IEA Greenhouse Gas Programme, E. S. Rubin, D. W. Keith, C. F.Gilboy (Eds.), Cheltenham, UK, pp. 83-91, 2005.

5 C. G. Van de Walle, J. Neugebauer, Nature 2003, 423, 626-628.

6 A. S. Nowick, A. V. Vaysleyb, Solid State Ionics 1997, 97, 17-26.

7 D. G. Thomas, J. J. Lander, J. Chem. Phys. 1956, 25, 1136-1142.

8 P. Kofstad, Non-Stoichiometry, Diffusion, and Electrical Conductivity in Binary Metal Oxides, Wiley, New York, 1972.

9 M. Widerøe, W. Münch, Y. Larring, T. Norby, Solid State Ionics 2002, 154 155, 669-677.

10 H. J. M. Bouwmeester, H. Kruidhof, A. J. Burggraaf, Solid State Ionics 1994, 72, 185.

11 K. F. Bonhoffer, F. Farkas, Trans. Faraday Soc. 1932, 28, 242.

12 A. Couper, D.D. Eley, Discuss. Faraday Soc. 1950, 8, 152.

13 A. Ozaki, F. Nozaki, K.-I. Maruya, S. Ogasawara, J. Catal. 1967, 7, 234.

14 Y. Amenomiya, J. Catal. 1971, 22, 109.

15 D. A. Dowden, N. Mackenzie, B. W. Trapnell, Proc. R. Soc. London, Ser.A 1956, 237, 245.

16 D. R. Ashmead, D. D. Eley, R. Rudham, J. Catal. 1964, 3, 280.

17 R. van de Krol, personal communication.

18 J. Maier, in Physical Chemistry of Ionic Materials: Ions and Electrons in Solids, Wiley, New York, 2004, pp. 338-362.
19 F.W. Poulsen, J. Solid State Chem. 1999, 143, 115-121.

20 S.-J. Song, E. D. Wachsman, J. Rhodes, S. E. Dorris, U. Balachandran, Solid State Ionics 2003, 164, 107-116.

21 J. B. Condon, T. Schober, Solid State Ionics 1997, 97, 51-58.

22 M. Saiful Islam, J. Mater. Chem. 2000, 10, 1027-1038.

23 W. Munch, K.-D. Kreuer, G. Seifert, J. Maier, Solid State Ionics 2000, 136137, 183-189.

24 T. Schober, J. Friedrich, Solid State Ionics 1999, 125, 319-323.

25 K.-D. Kreuer, E. Schoenherr, J. Maier, Solid State Ionics 1994, 70-71, 278-84.

26 F. Krug, T. Schober, R. Paul, T. Springer, Solid State Ionics 1995, 77, 185-188.

27 S. Marion, H.-W. Meyer, M. Carpenter, T. Norby, $\mathrm{H}_{2} \mathrm{O}-\mathrm{D}_{2} \mathrm{O}$ Exchange in Lawsonite, Am. Mineral. 2001, 86, 1166-1169.

28 K.-D. Kreuer, A. Fuchs, J. Maier, Solid State Ionics 1995, 77, 157-162.

29 N. Sakai, K. Yamaji, T. Horita, H. Yokokawa, Y. Hirata, S. Sameshima, Y. Nigara, J. Mizusaki, Solid State Ionics 1999, 125, 325-331.

30 E. Ruiz-Trejo, J. A. Kilner, Solid State Ionics 2000, 130, 313-324.

31 A. Groene, S. Kapphan, J. Phys. Chem. Solids 1996, 57, 325-331.

32 M. Glerup, F. W. Poulsen, R. W. Berg, Solid State Ionics 2002, 148, 83-92.

33 K. Amezawa, H. Maekawa, Y. Tomii, N. Yamamoto, Solid State Ionics 2001, 145, 233-240.

34 S. F. J. Cox, J. Phys.; Condens. Matter 2003, 15, R1727-1780.

35 R. Hempelmann, M. Soetratmo, O. Hartmann, R. Wappling, Solid State Ionics 1998, 107, 269-280.

36 K.-D. Kreuer, Th. Dippel, Yu.M. Baikov, J. Maier, Solid State Ionics 1996, 86-88, 613-620.

37 B. A. Boukamp, Solid State Ionics 1986, 18-19, 136-140.

38 Y. Larring, T. Norby, Solid State Ionics 1997, 97, 523-528.

39 R. Haugsrud, Y. Larring, T. Norby, Solid State Ionics 2005, 176, 2957-2961. 
40 R. Haugsrud, T. Norby, in Proc. $26^{\text {th }}$ Risø International Symposium on Material Science, S. Linderoth, A. Smith, N. Bonanos, A. Hagen, L. Mikkelsen, K. Kammer, D. Lybye, P. V. Hendriksen, F. W. Poulsen, M. Mogensen, W. G. Wang (Eds.), Risø National Laboratory, Roskilde, Denmark, pp. 209214, 2005.

41 T. Norby, Solid State Ionics 1988, 28-30, 1586-1591.

42 D. Sutija, T. Norby, P. Björnbom, Solid State Ionics 1995, 77, 167-174.

43 V.V. Kharton, F. M. B. Marques, Solid State Ionics 2001, 140, 381-394.

44 N. Bonanos, Solid State Ionics 2001, 145, 265-274.

45 T. Schober, J. Friedrich, D. Triefenbach, F. Tietz, Solid State Ionics 1997, 100, 173-181.

46 G. Hultquist, L. Gråsjö, Q. Lu, T. Åkermark, Corros. Sci. 1994, 36, 1459.

47 A. Ozaki, in Isotopic Studies of Heterogenous Catalysis, Kodansha Ltd., pp. 1-38, 1977.

48 E. Hörnlund, Appl. Surf. Sci. 2002, 199, 195.

49 S. Hamakawa, L. Li, A. Li, E. Iglesias, Solid State Ionics 2002, 48, 71.

50 H. Kruidhof, M. W. J. Luiten, N. E. Benes, H. J. M. Bouwmeester, in Carbon Dioxide Capture for Storage in Deep Geologic Formations-Results from the $\mathrm{CO}_{2}$ Capture Project, D. C. Thomas, S. M. Benson (Eds.), Elsevier, Vol. 1, pp. 365375, 2005.

51 H. Iwahara, T. Esaka, H. Uchida, N. Maeda, Solid State Ionics 1981, 3-4, 359.

52 H. Iwahara, H. Uchida, K. Ono, K. Ogaki, J. Electrochem. Soc. 1988, 135, 529-533.

53 H. Iwahara, T. Esaka, H. Uchida, T. Yamauchi, K. Ogaki, Solid State Ionics 1986, 18-19, 1003.

54 H. Iwahara, T. Yajima, T. Hibino, K. Ozaki, H. Suzuki, Solid State Ionics 1993, 61, 65.

55 A. Mitsui, M. Miyayama, H. Yanagida, Solid State Ionics 1987, 22, 213.

56 J. Guan, S.E. Dorris, U. Balachandran, M. Meilin, Ceram. Trans. 1999, 92, 265.

57 T. Fukui, S. Ohara, S. Kawatsu, J. Power Sources 1998, 71, 164.
58 T. Fukui, S. Ohara, S. Kawatsu, Solid State Ionics 1999, 116, 331.

59 L. Li, J. R. Wu, M. Knight, S. M. Haile, in Proc.-Electrochem. Soc., PV 2001-28 (Ionic and Mixed Conducting Ceramics), pp. 58-66, 2002.

60 K. A. Furøy, T. Norby, to be published.

61 U. Balachandran, T. H. Lee, G. Zhang, S. E. Dorris, K. S. Rothenberger, B. H. Howard, B. Morreale, A. V. Cugini, R. V. Siriwardane, J. A. Poston Jr., and E. P. Fisher, in Proceedings of the 26th International Technical Conference on Coal Utilization and Fuel Systems, Clearwater, FL, Mar. 5-8, 2001, Coal Technical Association, Gaithersburg, MD, pp. 751-761, 2001.

62 H. Matsumoto, T. Shimura, T. Hiuchi, T. Otake, Y. Sasaki, K. Yashioro, A. Kaimai, T. Kawada, J. Mizuzaki, Electrochemistry 2004, 72, 861.

63 H. Matsumoto, T. Shimura, T. Hiuchi, H. Tanaka, K. Katahira, T. Otake, T. Kudo, K. Yashioro, A. Kaimai, T. Kawada, J. Mizuzaki, J. Electrochem. Soc. 2005, 152, A488.

64 H. Matsumoto, T. Shimura, T. Hiuchi, T. Otake, Y. Sasaki, K. Yashioro, T. Kawada, J. Mizuzaki, S. Hashimoto, T. Ishihara, in Proceedings of the $26^{\text {th }}$ Risø International Symposium on Material Science, S. Linderoth, A. Smith, N. Bonanos, A. Hagen, L. Mikkelsen, K. Kammer, D. Lybye, P.V. Hendriksen, F.W. Poulsen, M. Mogensen, W.G. Wang (Eds.), Risø National Laboratory, Roskilde, Denmark, p. 285, 2005.

65 X. Qi, Y.S. Lin, Solid State Ionics 2000, 130, 149-156.

66 L. Li, E. Iglesia, Solid State Ionics 2003, 58, 1977.

67 G. C. Mather, M. Saiful Islam, Chem. Mater. 2005, 17, 1736.

68 E. D. Wachsman, J. Naixiong, U.S. Pat. 6235 417, May 22, 2001.

69 S. E. Dorris, T. H. Lee, U. Balachandran, U.S. Pat. 6569 226, May 27, 2003.

70 U. F. Reichel, Berichte des Forschungszentrums Jülich 1996, Jül-3318, pp. 1-142.

71 N. Sammes, R. Phillips, A. Smirnova, J. Power Sources 2004, 134, 153.

72 H. Uchida, H. Yoshikawa, T. Esaka, S. Ohtsu, H. Iwahara, Solid State Ionics 1989, 36, 89. 
73 W. Lian, Yi Ting, Z. X. Zhihong, J. Mater. Sci. Lett. 1994, 13, 1032.

74 T. Tsuji, T. Nagano, Solid State Ionics 2000, 136-137, 179.

75 S.-J. Song, E. D. Wachsman, J. Rhodes, S. E. Doris, U. Balachandran, J. Electrochem. Soc. 2003, 150, A1484.

76 S. Cheng, V. K. Gupta, Y. S. Lin, Solid State Ionics 2005, 176, 2653.

77 Y. Larring, personal communication.

78 A. N. Shirsat, K. N. G. Kaimal, S. R. Bharadwaj, D. Das, J. Solid State Chem. 2004, 177, 2007.

79 S. Okada, A. Mineshige, A. Takasaki, M. Kobunea, T. Yazawaa, H. Matsumoto, T. Shimura, H. Iwahara, Z. Ogumi, Solid State Ionics 2004, 175 , 593.

80 Y. Larring, T. Norby, Solid State Ionics 1994, 70-71, 305.

81 Y. Larring, PhD Dissertation; Protons and Oxygen Vacancies in Acceptor-Substituted Rare Earth Oxides, University of Oslo, 1998.

82 R. Haugsrud, Y. Larring, T. Norby, Solid State Ionics 2005, 176 (39-40), 29572961.

83 O. W. Johnson, S.-H. Paek, J. W. DeFord, J. Appl. Phys. 1975, 46, 1026.

84 R. Haugsrud, T. Norby, to be published.

85 R. Haugsrud, Y. Larring, to be published.

86 Y. Larring, R. Haugsrud, T. Norby, R. Bredesen, to be published.

87 T. Shimura, M. Komori and H. Iwahara, Solid State Ionics 1996, 86-88, 685-689.

88 J.A. Labrincha, J.R. Frade and F.M.B. Marques, Solid State Ionics 1997, 99, 33.

89 T. Shimura, Y. Tokiwa, H. Iwahara, Solid State Ionics 2002, 154-155, 653.

90 R. Haugsrud, T. Norby, Nature Materials 2006, 5, 193-196.

91 T. Shimura, S. Fujimoto, H. Iwahara, Solid State Ionics 2001, 143, 117.

92 R. Haugsrud, C. Kjølseth, T. Norby, to be published.

93 U. Balachandran, T. H. Lee, S. Wang, C. Zuo. S.E. Dorris, K.S. Rothenberger in Proceedings of the 20th International Pittsburgh Coal Conference, Pittsburgh, Sept. 15-19, 2003, S46, 187, Pittsburgh Coal Conference, University of Pittsburgh.
94 U. Balachandran, T. H. Lee, G. Zhang, S. E. Dorris, K. S. Rothenberger, B. H. Howard, B. Morreale, A. V. Cugini, R. V. Siriwardane, J. A. Poston Jr., E. P. Fisher, in Proceedings of the 26th International Technical Conference on Coal Utilization and Fuel Systems, Clearwater, FL, Mar. 5-8, 2001, Coal Technical Association, Gaithersburg, MD, pp. 751-761, 2001.

95 Y. Nigara, K. Kawamura, T. Kawada, J. Mizusaki, Solid State Ionics 2001, 145, 365-370.

96 Y. Nigara, K. Yashiro, T. Kawada, J. Mizusaki, Solid State Ionics 2003, 159, 135-141.

97 Y. Nigara, K. Yashiro, J.-O Hong, T. Kawada, J. Mizusaki, Solid State Ionics 2004, 171, 61-67.

98 E. Serra, A. Calza Bini, G. Cosoli, L. Pilloni, J. Am. Ceram. Soc. 2005, 88, 15-18.

99 T. Norby, P. Kofstad, High Temp.-High Press. 1988, 20, 345-360.

100 A. Bongiorno, L. Colombo, F. Cargnoni, Chem. Phys. Lett. 1997, 264, 435-440.

101 M.I. Heggie, R. Jones, C.D. Latham, S.C.P. Maynard, P. Tole, Philos. Mag. B, Phys. Condens. Matter 1992, 65, 463471.

102 M. Lacerda, A. R. West, J. T. S. Irvine, Solid State Ionics 1993, 59, 257-262.

103 K. Hayashi, M. Hirano, H. Hosono, J. Phys. Chem. B 2005, 109, 1190011906.

104 D. L. McKinley, U.S. Pat. 3439 474, Apr. 22, 1969.

105 D. J. Edlund, J. McCarthy, J. Membr. Sci. 1995, 107, 147.

106 F. Roa, J. D. Way, R. L. McCormick, S. N. Paglieri, J. Chem. Eng. 2003, 93, 11.

107 K. S. Rotheberger, B. H. Howard, R. P. Killmeyer, A. V. Cugini, R. M. Enick, F. Bustamante, M. V. Ciocco, B. D. Morreale, R. E. Buxbaum, J. Membr. Sci. 2003, 218, 19.

108 M. V. Mundschau, X. Xie, A. F. Sammells, in Carbon Dioxide Capture for Storage in Deep Geologic Formations Results from the $\mathrm{CO}_{2}$ Capture Project, D. C. Thomas, S. M. Benson (Eds.), Elsevier, Vol. 1, Ch. 16, pp. 291-306, 2005. 